\title{
Gentrifizierungsprozesse im Frankfurter Ostend. Stadtpolitische Aufwertungsstrategien und Zuzug der Europäischen Zentralbank
}

\author{
Andrea Mösgen ${ }^{1} \cdot$ Sebastian Schipper $^{1}$ (D)
}

Eingegangen: 25. Februar 2016 / Angenommen: 20. September 2016 / Online publiziert: 18. Oktober 2016

(C) Springer-Verlag Berlin Heidelberg 2016

Zusammenfassung Das innerstädtisch gelegene Ostend fungierte in Frankfurt am Main insbesondere seit den 1950er-Jahren als Entlastungsraum für den unteren Wohnungsteilmarkt und war dementsprechend von unterdurchschnittlichen Miet- und Bodenpreisentwicklungen geprägt. Da sich diese Entwicklung jedoch seit Mitte der 2000erJahre umkehrt, untersucht der Beitrag erstens, inwiefern der Stadtteil gegenwärtig von Gentrifizierung betroffen ist. Anhand sekundärstatistischer Quellen kann diesbezüglich gezeigt werden, dass in dem ehemaligen Arbeiterviertel vor allem exkludierende Verdrängungsprozesse wirken, was über die letzten 15 Jahre zu einer schleichenden, aber klar erkennbaren Veränderung der Sozialstruktur zugunsten höherer Einkommensgruppen geführt hat. Zweitens wird analysiert, wie das lange Zeit als nicht zu gentrifizieren geltende Viertel erfolgreich aufgewertet werden konnte. Dementsprechend argumentieren wir auf der Basis einer Dokumentenanalyse von Veröffentlichungen der Stadt Frankfurt, dass stadtpolitische Entscheidungsträger seit den 1980er-Jahren die Gentrifizierung des Ostends aktiv mittels diverser Instrumente ermöglicht und gefördert haben. Die dadurch ausgelösten Verdrängungsprozesse werden zudem durch den 2002 getroffenen Beschluss beschleunigt, den Sitz der Europäischen Zentralbank (EZB) ins Ostend zu verlegen. Anhand einer Diskursanalyse kann verdeutlicht

Dr. Andrea Mösgen

Moesgen@geo.uni-frankfurt.de

Dr. Sebastian Schipper

S.Schipper@geo.uni-frankfurt.de

1 Institut für Humangeographie, Campus Westend - PEG, Goethe-Universität Frankfurt am Main,

Theodor-W.-Adorno-Platz 6, 60629 Frankfurt am Main, Deutschland werden, dass die Umzugsentscheidung zu einer markanten symbolischen Aufwertung des Viertels geführt hat.

Schlüsselwörter Gentrification · Frankfurt am Main · Ostend · Verdrängung · Geldpolitik · Wohnungsmarkt · Europäische Zentralbank (EZB)

\section{Gentrification Processes in the Frankfurt Ostend District: Municipal Neighbourhood Upgrading Strategies and the Relocation of the European Central Bank}

Abstract The centrally located Ostend district in Frankfurt am Main had provided space for the lower-rent housing segment in particular since the 1950s and was therefore characterised by below-average rent price and real-estate value trends. Because these trends have reversed starting in the mid-2000s, this paper analyses at first the extent to which the Ostend district is impacted by gentrification. Secondary statistical sources show an exclusionary process of displacement in this former working class neighbourhood, which had led, during the last fifteen years, to a slow but clearly distinguishable change in the social structure favouring higher income groups. In a second step we analyse how the City of Frankfurt was able to upgrade the Ostend district - something that many had considered impossible. Through an analysis of municipal documents and publications we argue that municipal decision makers have used various tools for enabling and supporting the gentrification of this district since the 1980s. The subsequent displacement processes were accelerated by the 2002 decision to relocate the headquarters of the European Central Bank (ECB) to the Ostend district. Using discourse analysis we show that the reloca- 
tion decision has led to a distinctive symbolic valorization of the district.

Keywords Gentrification - Frankfurt - Ostend district . Displacement $\cdot$ Monetary policy $\cdot$ Housing market $\cdot$ ECB European Central Bank

\section{Einleitung}

Anfang der 2000er-Jahre entschied die Europäische Zentralbank (EZB), ihren Sitz ins Frankfurter Ostend zu verlegen. Seitdem ist der Stadtteil in den Fokus der medialen Aufmerksamkeit gerückt. Es wird befürchtet, dass dem traditionellen Arbeiterviertel nun die Gentrifizierung droht (z. B. Ochs 2002; Köneke 2015; Schulze 2015). Dies ist insofern bemerkenswert, da Bestrebungen der Stadt Frankfurt am Main, das Ostend umfassend aufzuwerten, keineswegs neu sind, sondern sich bis in die späten 1980er-Jahre zurückverfolgen lassen (Ronneberger/Keil 1995: 328 ff.). In Anbetracht dieser Situation untersucht der Beitrag erstens, inwiefern der Stadtteil gegenwärtig tatsächlich von Gentrifizierung betroffen ist, und fragt zweitens nach der Bedeutung von stadtpolitischen Aufwertungsstrategien einerseits sowie des Umzugs der Europäischen Zentralbank andererseits.

Entstanden ist das heute innerstädtisch gelegene Ostend mit 27.613 Einwohnern (Stadt Frankfurt am Main, Amt für Wohnungswesen 2015: 82; zur Lage vgl. Abb. 3) Mitte des 19. Jahrhunderts. Angesichts der ab den 1870er-Jahren im Zuge der Industrialisierung einsetzenden Wohnungsknappheit wurden in der östlichen Außenstadt Frankfurts sowohl Wohnhäuser für das Bürgertum als auch einfache Wohnungen für Arbeiterhaushalte errichtet. $\mathrm{Zu}$ einem industriell geprägten Arbeiterviertel entwickelte sich das Ostend ab der Jahrhundertwende mit der Einweihung des Osthafens 1912, dem Bau der Großmarkthalle in den 1920er-Jahren und der verkehrstechnischen Erschließung durch den Ausbau der Eisenbahn (Bischoff 2007: 196 ff.).

Im gesamtstädtischen Kontext Frankfurts fungierte das Ostend insbesondere seit dem Zweiten Weltkrieg als „Entlastungsraum für den unteren Wohnungsteilmarkt" (Ronneberger/Keil 1995: 329). Da der Wohnstandard vergleichsweise niedrig war, die Bausubstanz für Aufwertungsprozesse häufig unattraktiv erschien, Gleise, Durchgangsstraßen und Wallanlagen den Stadtteil vom Stadtzentrum trennen und selbigem zudem ein negatives Image ${ }^{1}$ anhing, konn-

\footnotetext{
1 Beispielsweise fragt die Frankfurter Neue Presse (FNP) im April 1959, ob bezüglich des vernachlässigten und verwahrlosten Zustands des Viertels ,der Osten Frankfurts zum ,Verbrecherviertel “ der Stadt geworden“ sei, und stellt fest, dass ,,die Frankfurter Vagabunden des Ostends fast ausnahmslos kriminell sind“ und in den ,Trümmerkellerkreisen [...] Bluttaten ausgeheckt“ würden (zitiert nach Bischoff 2007: 199 f.).
}

ten Menschen mit niedrigem Einkommen hier traditionell bezahlbaren Wohnraum finden. So schreibt etwa Freund (2002: 162): „Wegen seiner Nachbarschaft zu lärmbelästigenden Gewerbebetrieben mit Erwerbsmöglichkeiten für Gelegenheitsarbeiter (Großmarkthalle, Hafen) ist das südliche Ostend ein altes Durchgangsgebiet mit geringem Wohnwert und wies im Laufe der Jahrzehnte immer wieder Konzentrationen armer, gering integrierter und sozial schwacher Gruppen auf.“

Die historische Entwicklung als Arbeiterviertel, welches jahrzehntelang Haushalten mit niedrigen Einkommen, Tagelöhnern, Nichtsesshaften, Migranten und Arbeitslosen bezahlbaren Wohnraum bot, spiegelte sich lange Zeit in der relativ geringen Dynamik der Bodenpreise bzw. der Bodenrichtwerte ${ }^{2}$ wider. Wie in Abb. 1 dargestellt, sind die Bodenpreise im Ostend bis Mitte der 2000er-Jahre deutlich langsamer gestiegen als in anderen sowohl innenstadtnahen als auch peripheren Stadtvierteln. ${ }^{3}$ Unter der Annahme, dass Bodenpreise als kapitalisierte Grundrente zukünftige Ertragserwartungen reflektieren (Brede/Dietrich/Kohaupt 1976; Schipper 2013a), lässt sich Abb. 1 dahingehend interpretieren, dass Immobilieninvestoren noch bis vor gut zehn Jahren davon ausgingen, dass das Potenzial, im Ostend höhere Mieten durchsetzen zu können, trotz der zentralen Lage relativ gering ist.

$\mathrm{Ab}$ Mitte der 2000er-Jahre ändert sich dies jedoch grundlegend, insofern sich seitdem die Bodenpreisdynamik umgekehrt hat und das Ostend mit die höchsten Bodenpreissteigerungen in Frankfurt aufweist (vgl. Abb. 2). Diese Entwicklung deutet darauf hin, dass Immobilieninvestoren nun erwarten, im Ostend zukünftig höhere Mieten und Wohnungspreise erzielen zu können. Bezogen auf den Wohnungsbestand und existierende Mietverhältnisse lässt sich daraus schließen, dass in Relation zur gegenwärtigen Verwertung der Grundstücke, die noch durch vergleichsweise niedrige Mieten geprägt ist, eine Ertragslücke bzw. rent gap entstanden ist, die Gentrifizierungsprozesse antreibt (Clark 1995; Smith 1996; Schipper 2013a).

\footnotetext{
${ }^{2}$ Bodenrichtwerte bezeichnen den durchschnittlichen Lagewert von Grundstücken. Für Frankfurt am Main werden sie vom Gutachterausschuss für Immobilienwerte alle zwei Jahre auf der Grundlage einer Kaufpreissammlung flächendeckend ermittelt. Die Werte beziehen sich auf unbebaute Grundstücke, beziehen jedoch die von der Bauleitplanung vorgesehene Nutzungsart mit ein (Schipper 2013a: 189 ff.).

3 Untersucht wurde die Entwicklung der Bodenrichtwerte zwischen 1984 und 2016 für 102 ausgewählte Standorte im gesamten Frankfurter Stadtgebiet, bei denen sich im Zeitverlauf weder die Nutzung noch die zulässige Geschossflächenzahl verändert hat (zur Methode siehe Schipper 2013a). Zu Veranschaulichungszwecken werden in Abb. 1 und 2 Bodenpreisentwicklungen im Ostend mit Standorten in innerstädtischen sowie peripheren Stadtteilen verglichen. Zur besseren Lesbarkeit sind für die Abbildungen nur einige wenige Vergleichsstandorte ausgewählt worden, die als typisch für innerstädtische bzw. periphere Lagen gelten können.
} 


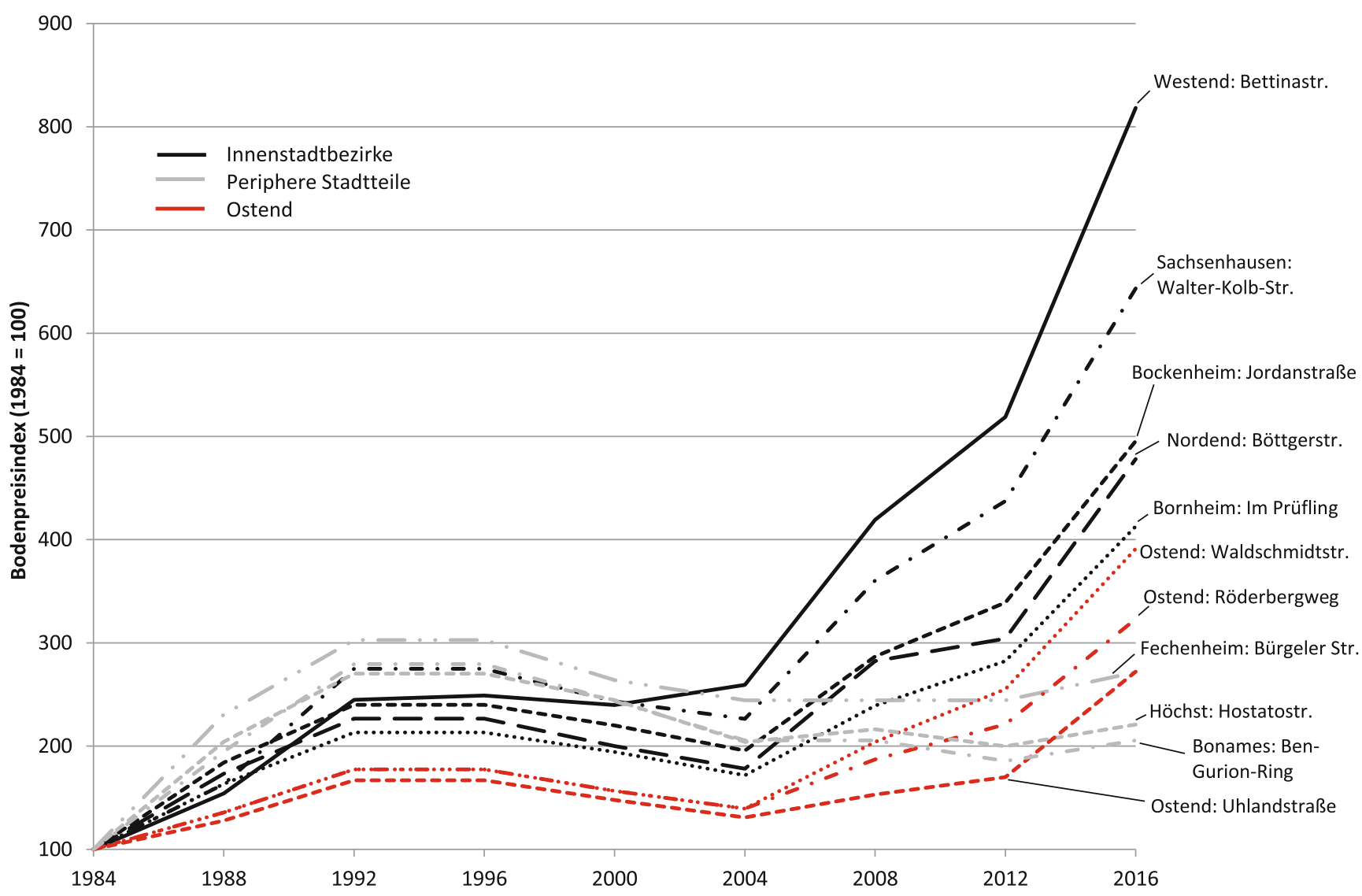

Abb. 1 Entwicklungsdynamik der Bodenrichtwerte an ausgewählten Standorten in Frankfurt am Main 1984 bis 2016,1984 = 100 (Daten: Gutachterausschuss für Immobilienwerte; eigene Berechnungen)

Gemäß der Rent gap-Theorie wird ein Stadtviertel für Immobilieninvestoren insbesondere dann interessant, wenn die Differenz zwischen tatsächlicher Grundrente, die sich aus den gegenwärtigen Mietzahlungen speist, und potenziell durchsetzbarer Grundrente, die durch die antizipierte profitabelste Verwertung eines Grundstückes bestimmt ist, steigt (Clark 1995; Smith 1996). Die abstrakten Kategorien der Ertragslücken-Theorie empirisch zu operationalisieren, ist jedoch mit gewissen Herausforderungen verbunden, da beispielsweise Mietzahlungen sowohl die Verzinsung des investierten Baukapitals als auch die Grundrente für die Verwertung des Bodens umfassen. Die in Abb. 1 und 2 betrachteten Bodenrichtwerte können jedoch als aussagekräftiger Indikator für die Entwicklung der potenziellen Grundrenten betrachtet werden, da sie für den jeweiligen Zeitpunkt die zukünftigen Ertragserwartungen für den reinen Boden unter Absehung des auf diesem investierten Kapitals widerspiegeln (Schipper 2013a). In Mietwohnungsmärkten verweist zudem eine ansteigende Differenz zwischen Bestands- und Angebotsmieten auf eine wachsende Ertragslücke und damit auf einen zunehmenden Verdrängungsdruck (Holm 2014: 282).

Vor diesem Hintergrund verfolgt der Beitrag zwei Ziele: Erstens untersuchen wir auf der Grundlage verschiedener sekundärstatistischer Quellen im folgenden Kap. 2, inwiefern sich die Schlussfolgerung einer ab Mitte der 2000erJahre entstandenen rent gap empirisch erhärten lässt und das Ostend tatsächlich gegenwärtig von Gentrifizierungsprozessen betroffen ist. Konzeptionell betrachten wir dabei die Verdrängung einkommensschwacher Schichten aus einem Wohnviertel als das Wesen der Gentrifizierung (Marcuse 1986). Gentrifizierung wird somit als der Prozess bestimmt, in dessen Verlauf ein „Austausch einer statusniedrigen Bevölkerung durch eine statushöhere Bevölkerung" (Friedrichs 1996: 14; ähnlich auch Holm 2014: 277) stattfindet. Unter dem Begriff der Verdrängung fassen wir in Anlehnung an die Typologie von Marcuse (1986) zum einen Formen der direkten Verdrängung, bei der Haushalte aufgrund von Mietsteigerungen im Bestand oder der Umwandlung in eine Eigentumswohnung gezwungen sind, ihren bisherigen Wohnstandort aufzugeben. Zum anderen sprechen wir von exkludierender Verdrängung, wenn in einem ehemaligen Arbeiterviertel mit traditionell relativ günstigen Mieten Schließungsprozesse stattfinden, sodass einkommensschwachen Haushalten der Zugang zu bezahlba- 


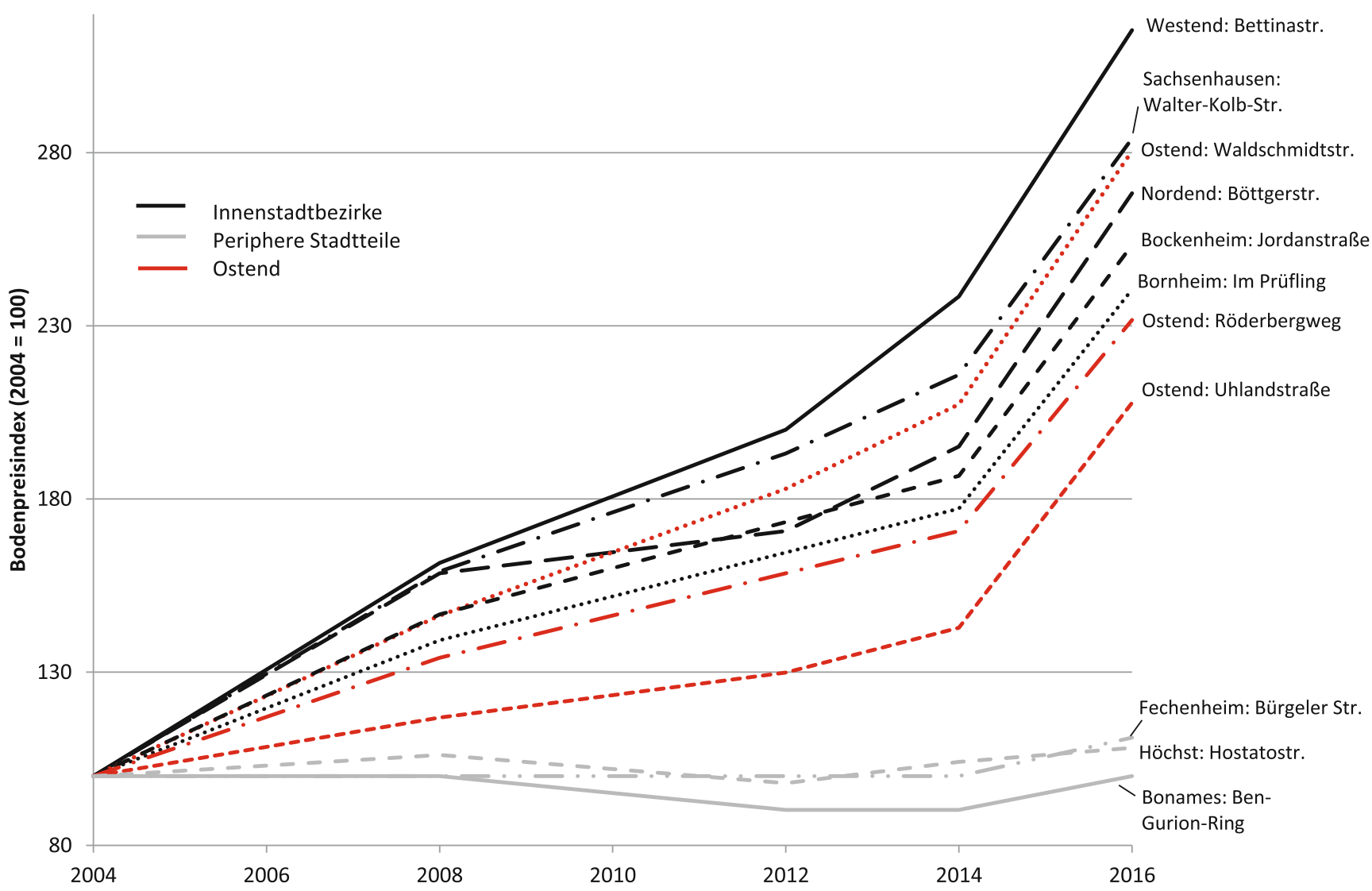

Abb. 2 Entwicklungsdynamik der Bodenrichtwerte an ausgewählten Standorten in Frankfurt am Main 2004 bis 2016 , 2004 = 100 (Daten: Gutachterausschuss für Immobilienwerte; eigene Berechnungen)

rem Wohnraum verwehrt wird. ${ }^{4}$ Zentraler Indikator hierfür ist die Entwicklung der Angebotsmieten. Zur Operationalisierung der ersten Fragestellung folgen wir dem Vorschlag von Davidson und Lees (2005: 1187), die vier Kernelemente der Gentrifizierung hervorheben: ,(1) the reinvestment of capital; (2) the social upgrading of locale by incoming high-income groups; (3) landscape change; and (4) direct or indirect displacement of low-income groups." Wie im Folgenden dargelegt wird, lassen sich alle vier Elemente seit Anfang bzw. Mitte der 2000er-Jahre im Ostend beobachten.

Zweitens werden wir analysieren, warum sich die Bodenpreisdynamik im Ostend ab Mitte der 2000er-Jahre so grundlegend umgekehrt hat und wie das Viertel erfolgreich aufgewertet werden konnte, sodass es nun zu Verdrängungsprozessen kommt. Diesbezüglich werden wir in Kap. 3 auf der Basis einer Dokumentenanalyse von Publikationen des Stadtplanungsamtes und Veröffentlichungen des Frankfur-

\footnotetext{
${ }^{4}$ Darüber hinaus kann man auch noch Formen der indirekten Verdrängung unterscheiden, bei denen sich die bisherigen Bewohner beispielsweise aufgrund von entfremdenden Veränderungen im Wohnumfeld zum Auszug entscheiden. Diese Art der Verdrängung kann aber mit den hier zur Anwendung kommenden Methoden nicht betrachtet werden.
}

ter Magistrats seit den 1980er-Jahren ${ }^{5}$ argumentieren, dass die Stadt Frankfurt die Gentrifizierung des Ostends mittels diverser Instrumente aktiv ermöglicht und gefördert hat. Darüber hinaus veranschaulicht Kap. 4, dass die Europäische Zentralbank (EZB) im Kontext dieser städtischen Aufwertungsmaßnahmen auf zweierlei Art und Weise Gentrifizierungsprozesse beschleunigt. Zum einen kann anhand einer Diskursanalyse der Lokalpresse ${ }^{6}$ verdeutlicht werden, dass der Umzug der Europäischen Zentralbank zu einer markanten symbolischen Aufwertung des Viertels geführt hat. Neben den lokalräumlichen Auswirkungen der Bank auf das Quartier hat zum anderen die extreme Lockerung der Geldpolitik als Reaktion auf die Finanz- und Schuldenkrise seit 2010 wesentliche strukturelle Voraussetzungen dafür

\footnotetext{
5 Im Rahmen der Dokumentenanalyse wurden sämtliche Veröffentlichungen der Stadt Frankfurt am Main bzw. konkreter des Magistrats, des Stadtplanungsamtes und des Ortsbeirats Bornheim/Ostend zu Planungsprozessen im Ostend untersucht. Genutzt wurde dazu unter anderem das Parlamentsinformationssystem (Parlis), welches es erlaubt, für den Zeitraum seit 1993 online nach Anfragen, Berichten, Stellungnahmen und Vorträgen des Magistrats, Debatten im Stadtparlament sowie Anträgen im Ortsbeirat zu recherchieren.

${ }^{6}$ Für den Zeitraum 2000-2015 wurden insgesamt 64 Artikel mit Bezug zum Ostend aus der Frankfurter Rundschau (FR) und der Frankfurter Allgemeinen Zeitung (FAZ) ausgewertet.
} 
Abb. 3 Mietpreise in den Frankfurter Stadtteilen 2014 und Änderung der Mietpreise von 2009 zu 2014 (Daten: IWU (2015: 49 ff.); eigene Berechnungen)

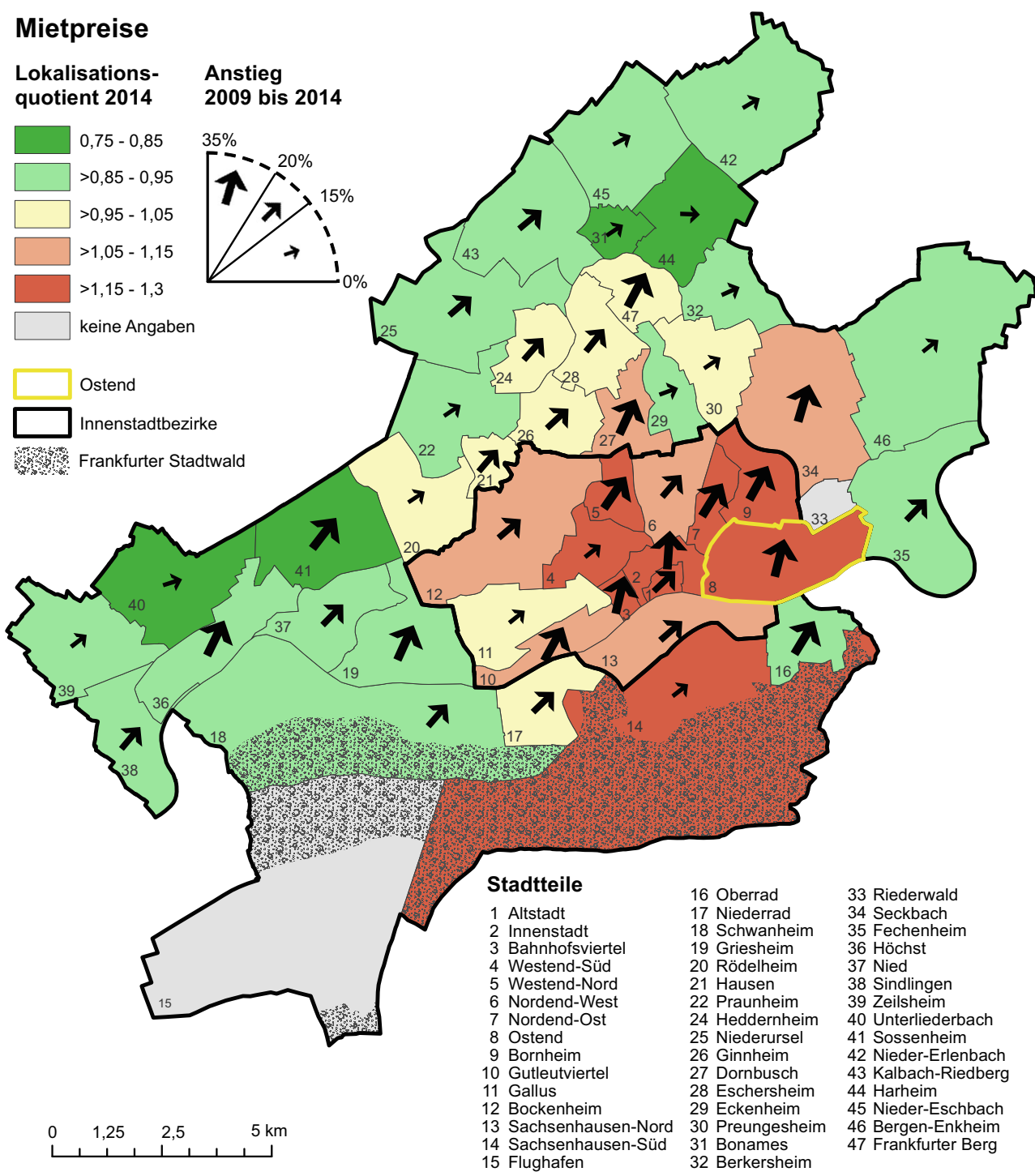

geschaffen, dass im Ostend, wie in vielen anderen Städten und Quartieren, Wohnungspreise und Mieten gestiegen sind. Angesichts sinkender Profitraten und niedriger Zinsen fließt nun verstärkt Kapital in den Wohnimmobilienmarkt, was ebenfalls Verdrängungsprozesse antreibt. Wesentliche Ergebnisse werden abschließend in Kap. 5 knapp zusammengefasst.

\section{Gentrifizierungsprozesse im Frankfurter Ostend}

Mit einer Einordnung in die gesamtstädtische Entwicklung wird im Folgenden anhand verschiedener sekundärstatistischer Indikatoren überprüft, ob sich im Ostend Gentrifizierungsprozesse beobachten lassen. Als Indikatoren des sozialstrukturellen Wandels und der Wohnungsmarktentwicklung dienen Daten zu Armut, Wohlstand und Miet- preisen. Armut wird hier über die Arbeitslosendichte ${ }^{7}$ sowie den Anteil an Empfängern von bedarfsorientierten Sozialleistungen ${ }^{8}$ abgebildet. Als Wohlstandsindikator werden die Einkommen herangezogen und anhand der Bruttoarbeitsentgelte sozialversicherungspflichtig Beschäftigter operationalisiert. $\mathrm{Zu}$ beachten ist, dass damit nicht alle Arten von (Erwerbs-)Einkommen der Bevölkerung erfasst werden. Ein umfassenderer Einkommensindikator steht jedoch nicht zur Verfügung. Mit der Verwendung dieser drei sozioökonomischen Indikatoren wird versucht, den sozialstrukturellen Wandel aus verschiedenen Perspektiven zu beleuchten, da kein Indikator die Armut oder den

\footnotetext{
7 Arbeitslosigkeit wird hier differenziert als Arbeitslosendichte, das heißt die Zahl der Arbeitslosen in Bezug auf die Bevölkerung im erwerbsfähigen Alter.

${ }^{8} \mathrm{Zu}$ den bedarfsorientierten Sozialleistungen zählen die Grundsicherung für Arbeitsuchende (ALG II) sowie im Alter und bei Erwerbsminderung, die Hilfe zum Lebensunterhalt und Asylbewerberleistungen.
} 
Wohlstand in einem Quartier alleine vollständig abdecken kann.

Während die Daten zur Bevölkerung aus der amtlichen Statistik stammen, stehen Mietpreise auf Stadtteilebene nur von privaten Anbietern zur Verfügung. Für die vorliegende Untersuchung wurde auf die Daten eines Gutachtens des Instituts für Wohnen und Umwelt (IWU) zurückgegriffen. Dieses im Auftrag der hessischen Landesregierung erstellte Gutachten dient der Feststellung von Gebieten mit angespannten Wohnungsmärkten und ist damit Grundlage für die Festlegung der sogenannten Mietpreisbremse in Hessen. Im Gutachten werden Daten des privaten Anbieters „IDN Immodaten" so aufbereitet, dass von zeitlich und räumlich konstanten Fehlern ausgegangen wird und damit Vergleiche zulässig sind (IWU 2015: 10). Ein Vergleich mit ebenfalls zur Verfügung stehenden Daten von „Immobilien Scout" bestätigt diese Annahme, da räumlich und zeitlich sehr ähnliche Muster festzustellen sind. Die verwendeten Daten aus dem IWU-Gutachten stellen Angebotsmieten in Euro/ $\mathrm{m}^{2}$ dar und bilden damit die jüngere Entwicklung auf dem Wohnungsmarkt ab. Für die meist etwas niedrigeren Bestandsmieten werden näherungsweise die Informationen des Mietspiegels herangezogen.

Für die Indikatoren Sozialleistungsbezug und Mieten stehen Daten einheitlich für den Zeitraum 2009 bis 2014 zur Verfügung. Für die Arbeitslosigkeit liegen darüber hinaus Daten seit 1997 vor, die für eine längerfristige Trendanalyse genutzt werden, um der Langsamkeit sozialstruktureller Prozesse Rechnung zu tragen. Einkommensdaten liegen für die Jahre 2005 und 2013 vor.

\subsection{Prozesse der exkludierenden Verdrängung}

Ausschlaggebend für exkludierende Verdrängungsprozesse auf dem Wohnungsmarkt sind die Angebotsmieten und ihre Entwicklung. Im Folgenden soll gezeigt werden, dass sich die in Kap. 1 dargestellten Trends steigender Bodenrichtwerte bereits in deutlich höheren Angebotsmieten niederschlagen, wodurch einkommensschwächere Haushalte im Ostend zunehmend vom Wohnungsmarkt ausgeschlossen werden. Im Zeitraum 2009 bis 2014 sind die Angebotsmieten in Frankfurt insgesamt um 20,2\% gestiegen, von 8,46 Euro auf 10,17 Euro je Quadratmeter (IWU 2015: 49).
Das Ostend liegt mit 29,3\% Steigerung nicht nur deutlich über dem Durchschnitt, sondern nach Innenstadt $(33,2 \%)$ und Bahnhofsviertel $(29,8 \%)$ an dritter Stelle im Stadtteilvergleich.

In Abb. 3 ist der Lokalisationsquotient der Angebotsmieten von 2014 dargestellt. Er wird berechnet als Relation der Stadtteilwerte zum städtischen Durchschnitt. Der Vorteil gegenüber der Abbildung absoluter Preise liegt in der Abstraktion von diesen je nach Datenanbieter leicht variierenden Werten, womit der oben diskutierten Problematik der Verwendung privater Daten begegnet wird. Stadtteile mit einem Lokalisationsquotienten über 1 sind demnach durch überdurchschnittliche Angebotsmieten gekennzeichnet, während Werte unter 1 auf unterdurchschnittliche Preise verweisen. Die Pfeile kennzeichnen die Mietsteigerung in Prozent.

Die höchsten Angebotsmieten und größten Zunahmen sind demnach nahezu flächendeckend in den innenstadtnahen Stadtvierteln zu verzeichnen. Ausnahmen sind lediglich einerseits das innenstadtnahe Gallusviertel mit durchschnittlicher Miethöhe und eher geringer Steigerung und andererseits die am Rand gelegenen Stadtteile Seckbach und Sachsenhausen-Süd mit überdurchschnittlichen Angebotsmieten. Das Ostend gehört 2014 zu den Stadtteilen mit den höchsten Angebotsmieten (11,75 Euro) und den höchsten Mietsteigerungen (29,3\%). Tab. 1 zeigt Höhe und Entwicklung der Angebotsmieten des Ostends im Vergleich mit den innenstadtnahen Stadtteilen (einschließlich Ostend) und äußeren Stadtteilen sowie den Werten für ganz Frankfurt. Insgesamt lassen sich zunehmende räumliche Gegensätze bei den Wohnkosten in Frankfurt feststellen. Die hohen und stark steigenden Angebotsmieten in den inneren Stadtteilen werden im Ostend übertroffen. In den äußeren Stadtteilen liegen die Angebotsmieten auf einem niedrigeren Niveau und wachsen langsamer.

Während das Ostend bei den Angebotsmieten zu den traditionell hochpreisigen Wohnlagen in Westend und Nordend aufgeschlossen hat, zeigt sich eine hohe Diskrepanz zu den Mieten im Bestand. Im Mietspiegel 2014, der als Indikator für die Bestandsmieten verwendet wird, gilt das Gebiet um den Ostbahnhof und südlich der Hanauer LandstraBe mit Ausnahme des direkten Mainufers als ,mittlere, einfache oder sehr einfache Wohnlage“" (Stadt Frankfurt 2014),

Tab. 1 Mieten und Sozialleistungsbezug im Ostend im Vergleich mit den inneren und äußeren Stadtteilen sowie dem Frankfurter Durchschnitt (Daten: Stadt Frankfurt am Main, Bürgeramt, Statistik und Wahlen; IWU (2015: 49 ff.); eigene Berechnungen)

\begin{tabular}{lllll}
\hline Gebiet & $\begin{array}{l}\text { Angebotsmiete 2014 } \\
\text { (in Euro) }\end{array}$ & $\begin{array}{l}\text { Entwicklung der Angebots- } \\
\text { miete 2009-2014 (in \%) }\end{array}$ & $\begin{array}{l}\text { Sozialleistungsbezieher } \\
\text { je 1000 Einwohner 2014 }\end{array}$ & $\begin{array}{l}\text { Entwicklung des Sozialleistungs- } \\
\text { bezugs 2009-2014 (in \%) }\end{array}$ \\
\hline Ostend & 11,75 & 29,3 & 116 & $-1,1$ \\
Innere Stadt $^{\mathrm{a}}$ & 11,66 & 21,6 & 116 & $-4,3$ \\
Äußere Stadt $^{\mathrm{a}}$ & 9,40 & 15,9 & 143 & 4,0 \\
Gesamtstadt & 10,17 & 20,2 & 130 & 0,8 \\
\hline
\end{tabular}

a Abgrenzung vgl. Abb. 3 und 5 
Abb. 4 Das Frankfurter Ostend (Quelle: Eigene Zusammenstellung auf Basis Stadt Frankfurt (2014) und von Daten vom Amt für Wohnungswesen der Stadt Frankfurt am Main)

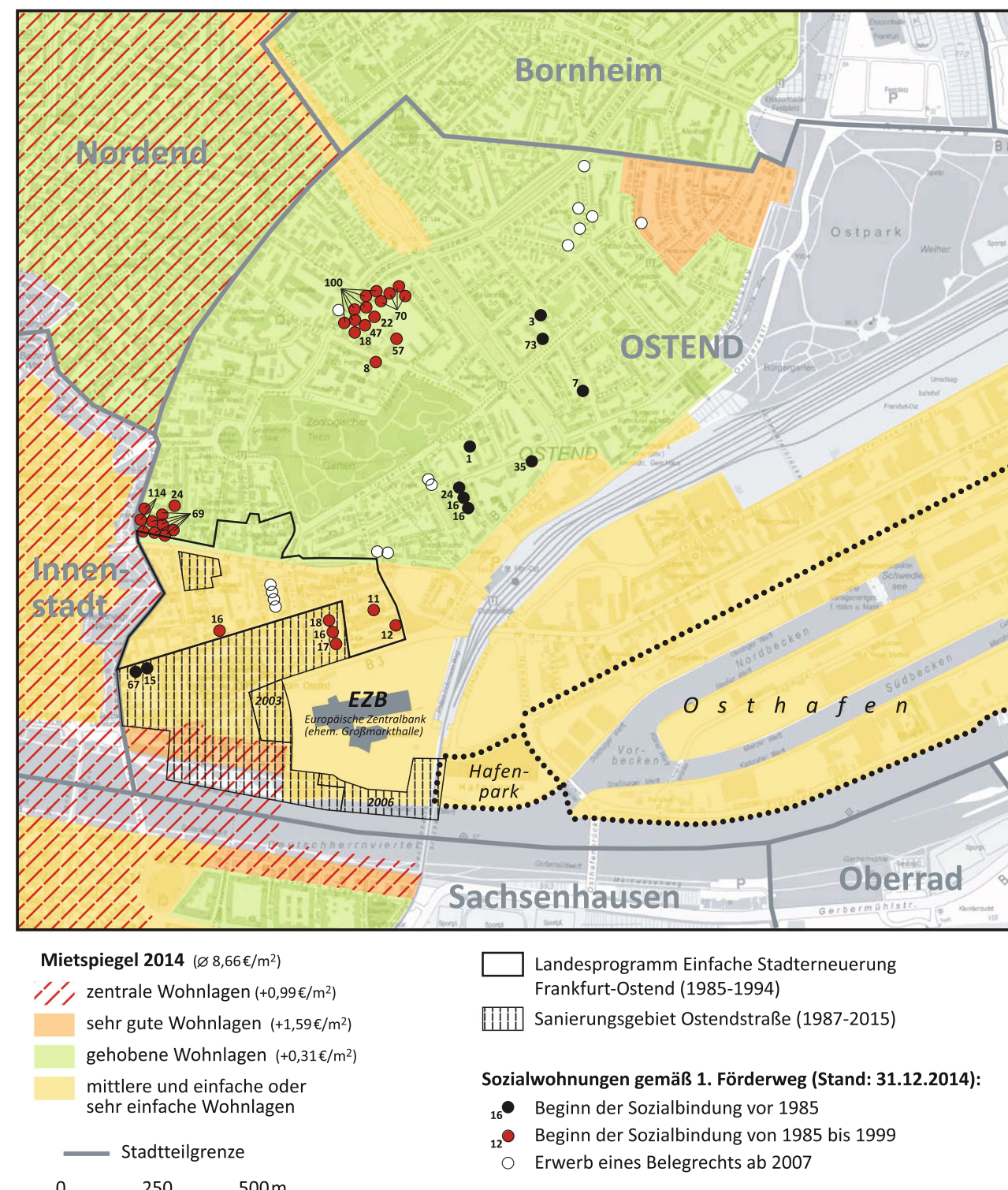

also als ein Gebiet, in dem aktuell noch preisgünstigere Wohnungen vorhanden sind (vgl. Abb. 4). Dieses Spannungsverhältnis zwischen überdurchschnittlich hohen Angebotsmieten und zum Teil noch relativ günstigen Bestandsmieten lässt sich als Ausdruck einer rent gap interpretieren. Die im Rahmen der Sanierungsmaßnahmen (vgl. Kap. 3) entstandene neue Wohnbebauung am Mainufer zählt dagegen entsprechend den Aufwertungszielen der Stadtpolitik bereits zu den „sehr guten Wohnlagen“, der höchsten Mietspiegel-Kategorie. Noch 2006 war dieses Gebiet als „einfache Wohnlage“ im Mietspiegel eingestuft. Mit Ausnahme der als „sehr gute Wohnlage“ eingestuften kleinen Fläche um den Parlamentsplatz liegen die übrigen Gebiete des Ostends als „gehobene Wohnlagen“ zwischen diesen Extremen. Aus diesem direkten Nebeneinander der Wohnlagen lässt sich ableiten, dass aktuell noch verschiedene Ein- kommensgruppen Wohnraum im Ostend finden, wobei die stark steigenden Preise einerseits und die Abnahme einkommensschwächerer Bevölkerungsgruppen andererseits (vgl. Kap. 2.2) darauf hinweisen, dass das preisgünstige Wohnungsmarktsegment rückläufig ist.

Im Ergebnis der Analyse der Veränderungen auf dem Wohnungsmarkt weisen die hohen Angebotsmieten und ihre starke Dynamik folglich auf Prozesse der exkludierenden Verdrängung hin. Für Wohnungssuchende mit niedrigen Haushaltseinkommen bietet das Ostend immer weniger bezahlbaren Wohnraum. Die länger ansässige Bevölkerung profitiert jedoch noch von den niedrigeren Bestandsmieten. 


\subsection{Veränderungen der Bevölkerungsstruktur als Hinweis auf direkte Verdrängungsprozesse}

Bei der direkten Verdrängung sind ansässige Haushalte gezwungen, ihre Wohnung aufgrund von Mietsteigerungen oder Umwandlung in Eigentumswohnungen aufzugeben. Prozesse der direkten Verdrängung von Bevölkerungsgruppen sind auf der Grundlage der Sekundärstatistik nicht explizit erfassbar, da die Daten nichts über die Art des Prozesses und auch nichts über Kausalitäten aussagen. Gleichzeitig stattfindende Prozesse der Wohnraumverteuerung und des Rückgangs einkommensschwacher Bevölkerungsgruppen können jedoch als starke Hinweise auf Verdrängung gedeutet werden. Um in diesem Sinne die Veränderung der Bevölkerungsstruktur zu analysieren, werden im Folgenden neben den Bruttoarbeitsentgelten der sozialversicherungspflichtig Beschäftigten auch Sozialleistungsbezug und Arbeitslosigkeit untersucht, um sowohl Wohlstand als auch Armut abbilden zu können.

Der Sozialleistungsbezug ist im Frankfurter Durchschnitt zwischen 2009 und 2014 geringfügig von 12,9\% auf 13,0\% gestiegen. Im Ostend ist dagegen mit $-1,1 \%$ ein leichter Armutsrückgang zu verzeichnen. Die folgende Karte des Sozialleistungsbezugs und seiner Entwicklung (Abb. 5) zeigt ein weniger offensichtliches Muster als die Karte der Mieten (Abb. 3). Es lässt sich jedoch festhalten, dass in den innenstadtnahen Stadtteilen unterdurchschnittliche oder durchschnittliche Armutsquoten überwiegen und dass diese weiter abnehmen (vgl. auch Tab. 1). Ausnahmen sind das Gallusviertel mit einer hohen, aber sinkenden Armut sowie die Altstadt mit steigender Armutsquote. Überdurchschnittliche Armut ist in (ehemals) industriell geprägten Stadtteilen entlang des Mains zu verzeichnen. Außerdem fällt der steigende Sozialleistungsbezug in zahlreichen peripheren Stadtteilen auf. Zusammenfassend zeigt Tab. 1 geringere und abnehmende Armut in den inneren Stadtteilen, während die äußeren Stadtteile durch höheren und steigenden Sozialleistungsbezug gekennzeichnet sind. Das Ostend weist eine unterdurchschnittliche Armutsquote bezüglich der Gesamtstadt auf und liegt genau im Durchschnitt der inneren Stadtteile. Der Sozialleistungsbezug ist rückläufig, aber schwächer als in den anderen innerstädtischen Gebieten.

Beim Vergleich der räumlichen Muster der Miet- und Armutsentwicklung9 ${ }^{9}$ sind Stadtteile mit überdurchschnittlichen Mietsteigerungen und zugleich einem Armutsrückgang tendenziell als Stadtteile anzusehen, in denen die Gefahr von Verdrängung vorliegt. Dagegen sind Stadtteile

\footnotetext{
${ }^{9}$ In diesem Kapitel sollen keine statistischen Zusammenhänge dargestellt werden, sondern inhaltliche Annahmen dergestalt, dass das $\mathrm{Zu}$ sammentreffen von starker Mietsteigerung und gleichzeitig starkem Rückgang der Armut auf Verdrängungsgefahr hinweist.
}

mit zunehmender Armut potenziell als Zielgebiete von verdrängter Wohnbevölkerung anzunehmen (z. B. Fechenheim, Harheim, Zeilsheim), sofern diese nicht aus Frankfurt ins Umland wegzieht. Häufig liegen diese Stadtteile eher am Stadtrand und sind bereits durch höhere Armut sowie niedrigere Mieten geprägt.

Im Ostend weist der trotz der hohen und überaus stark steigenden Angebotsmieten nur leichte Armutsrückgang kaum auf direkte Verdrängung hin. Allerdings liegt die Entwicklung im Ostend mit $-1,1 \%$ klar unter dem städtischen Durchschnitt von $+0,8 \%$ (vgl. Tab. 1). Ein möglicher Grund für den 2009 bis 2014 nur wenig rückläufigen Sozialleistungsbezug im Ostend ist, dass die Stadt Frankfurt die Kosten der Unterkunft für SGBII ${ }^{10}$-Empfänger bis zur Höhe der ortsüblichen Vergleichsmiete trägt. Das heißt, die Stadt Frankfurt fängt steigende Bestandsmieten auf, sodass im Ostend wohnhafte Sozialleistungsbezieher über Individualförderung derzeit vor Verdrängung geschützt sind, was für Geringverdiener ohne staatliche Unterstützung jedoch nicht gilt. Zudem bietet der Frankfurter Wohnungsmarkt kaum Ausweichmöglichkeiten für von Mietsteigerungen betroffene Haushalte, da die Mieten in Frankfurt insgesamt hoch sind und flächendeckend steigen (IWU 2015: 50). Zu vermuten ist daher, dass einkommensschwache Haushalte im Falle steigender Bestandsmieten ihre Wohnung nicht aufgeben, sondern einen höheren Anteil ihres Einkommens für Wohnkosten verwenden, was letztlich zu einer Verdrängung aus dem Lebensstandard führt.

Ein Blick auf längere Zeiträume und andere Indikatoren, wie Arbeitslosigkeit und Einkommen, die im Folgenden betrachtet werden, bestätigt, dass die Armut im Ostend abgenommen hat. Da der Sozialleistungsbezug aufgrund definitorischer Änderungen nicht langfristig rekonstruierbar ist, wird hierfür die Arbeitslosendichte herangezogen, für die Daten seit 1997 auf Stadtteilebene existieren. Wie Abb. 6 zeigt, war das Ostend lange Zeit durch überdurchschnittlich hohe Arbeitslosigkeit gekennzeichnet. Parallel zur Umsetzung der Aufwertungsmaßnahmen (vgl. Kap. 3) haben sie sich allmählich dem städtischen Durchschnitt angenähert, seit 2006 liegen sie leicht darunter. Der Wandel der Bevölkerungsstruktur vollzieht sich im Ostend also in einem langfristigen Prozess.

Diese Tendenz abnehmender Armut bezüglich Sozialleistungsbezug und Arbeitslosigkeit wird durch einen $\mathrm{Zu}$ zug wohlhabenderer Personen mit höheren Erwerbseinkommen verstärkt. So haben die Bruttoarbeitsentgelte der sozialversicherungspflichtig Beschäftigten zwischen 2005 und 2013 im Ostend überdurchschnittlich zugenommen: Der Median erhöhte sich um 22,1 \%, während er in ganz Frank-

\footnotetext{
${ }^{10}$ Sozialgesetzbuch II: Grundsicherung für Arbeitsuchende.
} 
Abb. 5 Sozialleistungsbezug in den Frankfurter Stadtteilen 2014 und Änderung des Sozialleistungsbezugs $2009 \mathrm{zu}$ 2014 (Daten: Stadt Frankfurt am Main, Bürgeramt, Statistik und Wahlen; eigene Berechnungen)

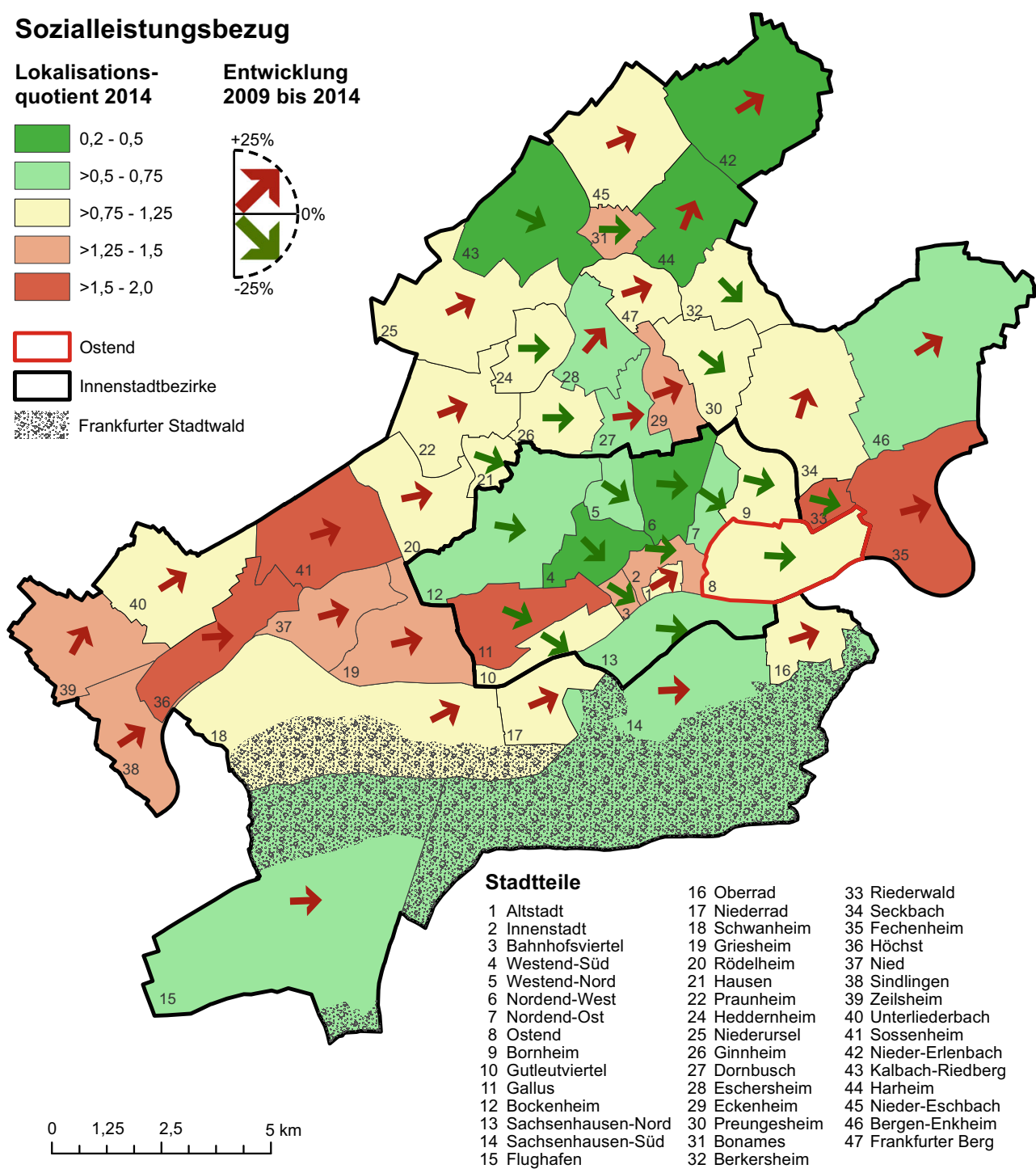

furt um $17,1 \%$ stieg. ${ }^{11}$ In der Folge lagen die Einkommen 2013 mit 3.750 Euro deutlich über dem Frankfurter Durchschnitt von 3.579 Euro. ${ }^{12}$

Als Zwischenfazit lassen sich Gentrifizierungsprozesse im Ostend festhalten. Dies gilt insbesondere bezüglich der exkludierenden Verdrängung, da hohe und weiter stark steigende Angebotsmieten zu Schließungsprozessen auf dem Wohnungsmarkt führen. Direkte Verdrängungsprozesse können dagegen auf der Grundlage der sekundärstatistischen Analysen nicht explizit nachgewiesen werden. Allerdings hat sich in den letzten 15 Jahren die Bevölke-

\footnotetext{
${ }_{11}$ Daten vom Bürgeramt, Statistik und Wahlen, Stadt Frankfurt am Main.

12 Zur Einschätzung der Einkommenshöhe ist festzuhalten, dass nur die Bruttoeinkommen von Vollzeiterwerbstätigen und sozialversicherungspflichtig beschäftigten Personen erfasst werden. Mögliche Verzerrungen beziehen sich jedoch auf alle Frankfurter Stadtteile, sodass die Relationen bestehen bleiben und Vergleiche möglich sind.
}

rungsstruktur im Ostend langsam, aber klar erkennbar gewandelt, indem Sozialleistungsbezug und Arbeitslosigkeit rückläufig sind und gleichzeitig die Erwerbseinkommen überdurchschnittlich steigen. Die festgestellte Ertragslücke (rent gap) zu den Bestandsmieten im südlichen Teil des Ostends lässt weitere Sanierungen mit Mietsteigerungen oder Umwandlung in Eigentumswohnungen erwarten, wodurch die direkte Verdrängung und der Bevölkerungsaustausch zugunsten einkommensstärkerer Schichten vermutlich zunehmen werden. Auf der Grundlage dieser Diagnose beleuchten wir im folgenden Kapitel, inwiefern stadtpolitische Aufwertungsstrategien seit Ende der 1980er-Jahre eine solche Verdrängungsdynamik in Gang gesetzt haben. 


\section{Stadtpolitische Strategien der Aufwertung im Ostend}

„So geschieht die Aufwertung des Ostends nicht natürlich [...]. Was sich im Ostend tut, ist zuallererst die Folge eines großen Plans der Stadt“" (Nienhaus 2013: 25).

Spätestens seit Ende der 1980er-Jahre ist die Stadt Frankfurt am Main im Kontext der "Global-City-Formierung“ (Ronneberger/Keil 1995; Schipper 2013a) und ihrer unternehmerischen Ausrichtung (Schipper 2013b) bestrebt, das Ostend als Standort für ,verdichteten Wohnungsbau von hoher Wohnqualität“" (Stadt Frankfurt 1994: 6) aufzuwerten (Ronneberger/Keil 1995: 328 ff.). Aus der Perspektive der Frankfurter Stadtregierung bestand damals angesichts ,immer deutlicher werdender gravierender Missstände" (Stadt Frankfurt 2015: 7) ein dringender stadtplanerischer Handlungsbedarf ,zur Umkehr dieser negativen Entwicklung“, da „,das Ostend im Vergleich zu den umliegenden Stadtteilen qualitativ eine geringere wirtschaftliche Aktivität, Wohnqualität und Investitionstätigkeit“" (Stadt Frankfurt 2015: 7) aufweise. Als Entwicklungsziele definierte die Stadt, emissionsstarke Gewerbebetriebe zu verlagern, die Wohnqualität im Viertel zu stärken und die Stadtgestalt insgesamt aufzuwerten (Stadt Frankfurt 2015: 11 f.). Tiefgreifende staatliche Eingriffe zur Initiierung von derartigen Aufwertungsprozessen erschienen den städtischen Entscheidungsträgern als notwendig, weil trotz der innenstadtnahen Lage eine ,aus dem Stadtteil heraus eigenständig motivierte Entwicklung nicht abzusehen" (Stadt Frankfurt 2015: 8) sei, private „Investitionen seitens der Hauseigentümer" ausblieben und auf „,den Grundstücken innerhalb des Untersuchungsgebietes [...] kein Verwertungsdruck“" (Stadt Frankfurt 2015: 8) bestünde.

Vor diesem Hintergrund wurde daher 1985 eine „Vorbereitende Untersuchung" im Ostend durchgeführt, die im Ergebnis in einer förmlichen Festsetzung des Bereichs südlich der Ostendstraße als Sanierungsgebiet (21,16 ha) mündet (Abb. 4). ${ }^{13}$ Die Umsetzung der Sanierung beginnt daraufhin im Frühjahr 1987, wobei die letzten Schritte Ende 2015, also knapp 30 Jahre später, abgeschlossen werden (vgl. Kap. 3.2). Die städtebaulichen Missstände im Gebiet nördlich der Ostendstraße (14,3 ha) sollen dagegen mit Maßnahmen geringerer Intensität im Rahmen des Landesprogramms „Einfache Stadterneuerung“ (1985-1994) behoben werden (vgl. Kap. 3.1). Während die Pläne zur Restrukturierung des Stadtteils anfangs aufgrund finanzieller Schwierigkeiten und Widerständen bei der Gewerbeverlagerung relativ schleppend anlaufen (Ronneberger/Keil

\footnotetext{
13 Das Gebiet von ursprünglich 18,66 ha wird 2003 um 1,02 ha und 2006 um 1,48 ha erweitert.
}

1995: 329 f.), ${ }^{14}$ zeichnet sich ab Mitte der 2000er-Jahre ein grundlegender Wandel ab. Grund ist zum einen die Entscheidung von 2002, den Sitz der Europäischen Zentralbank ins Ostend zu verlagern, was unter anderem zu einer symbolischen Aufwertung des Viertels führt (vgl. Kap. 4), sowie zum anderen die Tatsache, dass die beiden tiefgreifenden städtebaulichen Sanierungs- und Erneuerungsprogramme nun ihre Wirkung zu entfalten beginnen. Bemerkenswert an den beiden Programmen ist zudem, dass die frühzeitig durchgeführten Aufwertungsmaßnahmen bis Ende der 1990er-Jahre noch von Instrumenten flankiert waren, die Verdrängungsprozesse verhindern sollten. Bei den späteren Sanierungsschritten ab 2000 ist dies nicht mehr der Fall.

\subsection{Aufwertung (fast) ohne Verdrängung: „Einfache Stadterneuerung“ 1985-1994}

Ziel des auf zehn Jahre angelegten Programms der „Einfachen Stadterneuerung“ (1985-1994) war es, ,mit mittlerem Aufwand im öffentlichen Bereich und Anstoßwirkung im privaten Bereich die Verbesserung von Gebieten, die einzelne städtebauliche Mängel oder Mißstände aufweisen, zu fördern“" (Bischoff 2007: 194). Konkret sollte die Wohnnutzung etwa durch die öffentliche Bezuschussung von baulichen Sanierungsmaßnahmen gestärkt, störende Gewerbebetriebe verlagert und die Verkehrssituation verbessert werden (Stadt Frankfurt 1994: 6 f.).

Bemerkenswert ist der noch sozialverträgliche Charakter der ,Einfachen Stadterneuerung'. Laut dem Abschlussbericht des Programms aus dem Jahre 1994 war wesentliches Entwicklungsziel, die „Versorgung des unteren Wohnungsteilmarktes“" sicherzustellen und eine „Verdrängung der derzeitigen Bewohner" zu vermeiden (Stadt Frankfurt 1994: 8). Dazu galt es erstens, mit öffentlichen Fördermitteln private Eigentümer von der Instandsetzung der vorhandenen Bausubstanz bzw. von einer „Modernisierung mit einfachen Standards“ (Stadt Frankfurt 1994: 8) zu überzeugen und dabei im Gegenzug eine temporäre Mietbindung von 6,00 bis maximal 9,50 DM/m² über 12 Jahre, einen Verzicht auf die Umlage der Modernisierungskosten auf die Miete sowie ein Umwandlungsverbot in Eigentumswohnungen vertraglich festzuschreiben (Stadt Frankfurt 2015: 20). Durch die Bereitstellung von 7,6 Mio. DM an Fördermitteln durch das Land Hessen und weiterer 1,8 Mio. DM durch die Stadt Frankfurt konnten schließlich im gesamten Gebiet bis 1994 etwa 300 Wohneinheiten (davon 128 mit öffentlicher För-

\footnotetext{
${ }^{14} \mathrm{Zu}$ dieser Zeit erhält das Ostend mit einem S-Bahn- (1988) und U-Bahn-Anschluss (1992) eine verkehrliche Aufwertung, was laut Aussage des Stadtplanungsamtes ,,auch negative Folgen wie Bodenpreissteigerungen und beginnende Tendenzen von Bodenspekulationen zur Folge" (Stadt Frankfurt 2015: 9) gehabt habe.
} 
Abb. 6 Entwicklung der Arbeitslosendichte des Ostends im Vergleich zu Frankfurt insgesamt 1997 bis 2014 (Daten: Stadt Frankfurt am Main, Bürgeramt, Statistik und Wahlen; eigene Berechnungen)

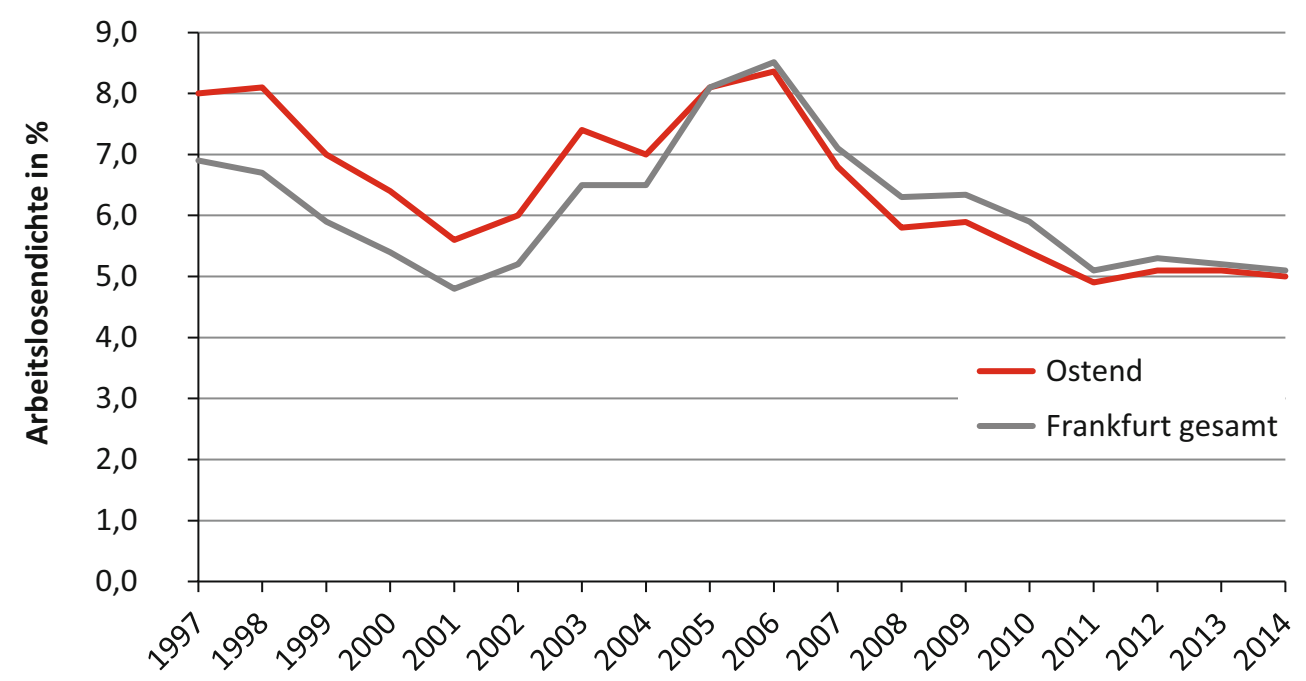

derung) saniert werden. Der volle Umfang der bewilligten Mittel wurde jedoch nicht ausgeschöpft, da die Mehrheit der in Frage kommenden Hauseigentümer nicht an einer Modernisierung ihrer Gebäude interessiert war, weil sie die Bewilligungsmiete als zu niedrig beurteilte. Eine höhere Bewilligungsmiete wollte aber die Stadt Frankfurt wiederum nicht zulassen, da „höhere Mieten [...] möglicherweise zu Verdrängungsprozessen geführt“ (Stadt Frankfurt 1994: 22) hätten.

Zudem hatten zweitens „Neubaumaßnahmen in Baulücken und auf brachliegenden Flächen vorrangig im Rahmen des sozialen Wohnungsbaus" (Stadt Frankfurt 1994: 8) zu erfolgen. Dementsprechend entstanden 1990 bei vier Neubauprojekten im Gebiet der „Einfachen Stadterneuerung" ausschließlich Sozialwohnungen gemäß dem 1. Förderweg (insgesamt 47 Wohneinheiten). Da die Wohnbauförderung damals überwiegend mit relativ langen Bindungsfristen operiert hat (im Unterschied zu späteren Wohnbauförderprogrammen, vgl. folgendes Kapitel), befinden sich auch Ende 2014 noch immerhin 39 der damals errichteten Sozialwohnungen weiterhin in der Preis- und Belegungsbindung (vgl. die drei einzelnen roten Punkte 11, 12 und 16 im Gebiet der „Einfachen Stadterneuerung“ in Abb. 4).

\subsection{Städtebauliche Sanierung „Ostendstraße“ im südlichen Ostend 1987-2015}

Südlich der Ostendstraße führt die Stadt Frankfurt zwischen 1987 und 2015 eine im Vergleich zur „Einfachen Stadterneuerung“" weit umfassendere „Städtebauliche Sanierungsmaßnahme“ durch, um aus „einem ehemals gewerblich-industriell geprägten Gebiet [...] ein attraktives Stadtquartier“ (Stadt Frankfurt 2007: 6) zu machen (vgl. Abb. 4). Genutzt werden dazu neben öffentlichen Fördermitteln die weitreichenden bodenrechtlichen Eingriffsmöglichkeiten, welche das Baugesetzbuch vorsieht. In dem Sanierungsgebiet entstehen ab 1994 und verstärkt ab 2000 insgesamt etwa 1000 neue Wohneinheiten.

Als Entwicklungsziel wird seitens der Stadt neben der Behebung städtebaulicher Missstände und der Aufwertung des Stadtteils zu Beginn des Verfahrens ebenfalls hervorgehoben, dass eine „Verdrängung der alteingesessenen Bewohner" vermieden werden müsse und es wichtig wäre, „die Bedeutung des Quartiers für den unteren Wohnungsteilmarkt in Form von gefördertem Wohnungsbau weiterhin zu bewahren" (Stadt Frankfurt 2015: 11 f.). Wie im Folgenden dargelegt wird, lässt sich im Kontext der städtebaulichen Sanierung jedoch ab der Jahrtausendwende ein grundlegender Bruch bezüglich der Zielrichtung identifizieren. Im Gegensatz zum Programm der „Einfachen Stadterneuerung" und im Unterschied zu den frühen Sanierungsmaßnahmen zwischen 1987 und 1999 werden die Aufwertungsbestrebungen und umfangreichen Bauvorhaben ab spätestens Anfang der 2000er-Jahre nicht mehr von Instrumenten begleitet, die Verdrängungsprozesse verhindern (sollen).

Um das Ziel der Aufwertung des Stadtteils zu erreichen, verfolgt die Stadt im Rahmen der Städtebaulichen Sanierung „Ostendstraße“ im Wesentlichen vier Strategien: Erstens werden die öffentlichen Plätze im südlichen Ostend renoviert, ehemalige Hafenflächen grundlegend umgestaltet und zahlreiche attraktive Grünflächen, Spielplätze und Freizeitanlagen neu geschaffen (beispielsweise der Mainuferpark sowie der außerhalb des Sanierungsgebiets liegende Hafenpark). Zudem werden, zweitens, zahlreiche Kulturund Bildungsinstitutionen neu angesiedelt. Dazu zählen etwa eine private Hochschule der Finanzindustrie, der Wiederaufbau der Alten Stadtbibliothek sowie das Bildungszentrum Ostend, welches unter anderem die Volkshochschule, ein Abendgymnasium und eine Berufsschule umfasst. Drittens bewirkt die Stadt eine Verlagerung des störenden Kleingewerbes mit hoher Lärm- und Emissionsbelastung 
und nimmt, viertens, im südlichen Ostend eine umfangreiche und großflächige Neuordnung mindergenutzter Flächen vor (Stadt Frankfurt 2015).

Um die kleinteilige Eigentümerstruktur südlich der Sonnemannstraße und westlich der Großmarkthalle aufzubrechen und attraktive Grundstückszuschnitte für gehobenen Wohnraum zu schaffen, hat die Stadt für insgesamt 43 Mio. Euro Flächen aufgekauft, Gebäude abgerissen, Altlasten entsorgt und Entschädigungen gezahlt mit dem Ziel, die so neugeordneten Grundstücke anschließend wieder an private Investoren zu verkaufen (Stadt Frankfurt, Stadtplanungsamt 2007; Stadt Frankfurt 2015). Die Verkaufserlöse aus den neugeordneten und dann reprivatisierten Grundstücken sollten laut Auftrag des Magistrats zudem dafür sorgen, dass die Aufwertungsmaßnahmen ,sich selbst finanzier[en]" (Magistrat der Stadt Frankfurt 2003: 2). Im Unterschied zur „Einfachen Stadterneuerung“ wird so ab Frühjahr 2000 in der entscheidenden Umsetzungsphase der städtebaulichen Sanierung ein Mechanismus verankert, welcher strukturell Maßnahmen zur Verhinderung von Verdrängungsprozessen entgegensteht, da er dafür sorgt, dass die städtischen Entscheidungsträger selbst ein Interesse an hohen Bodenpreisen und einem entsprechend hohen Mietniveau entwickeln. Städtebauliche Fördermittel zur Verhinderung von Verdrängungsprozessen oder Mietpreisbindungen sind dagegen nicht mehr vorgesehen. Während zwischen 1987 und 1999 die Sanierung noch federführend durch das Stadtplanungsamt bearbeitet wurde, führten die Reduzierung staatlicher Fördermittel und ein Personalabbau im Stadtplanungsamt zudem dazu, dass die Trägerschaft an eine private Agentur ausgelagert wird (Stadt Frankfurt 2015: 15). Um „,das Verfahren finanziell zu entlasten“ (Magistrat der Stadt Frankfurt 2015: 2), setzt die Stadt zur Erreichung der oben genannten Ziele ab März 2000 die Deutsche Bau- und Grundstücks-AG als treuhänderischen Sanierungsträger ein, die sich seitdem vorrangig um den Verkauf der städtischen Liegenschaften kümmert (Magistrat der Stadt Frankfurt 2003).

Nach Abschluss des Sanierungsverfahrens im September 2015 stehen den Gesamtkosten von 68 Mio. Euro schließlich 44 Mio. Euro an Einnahmen durch die Privatisierung städtischer Liegenschaften und die Erhebung von Ausgleichsbeträgen sowie 13 Mio. Euro an Zuschüssen von Bund und Land gegenüber. Bei der Stadt verbleibt so trotz der hochpreisigen Vermarktung der restrukturierten Grundstücke ein Defizit von 11,3 Mio. Euro (Stadt Frankfurt 2015: 76 ff.). Insgesamt sind im Sanierungsgebiet und dort hauptsächlich südlich der Sonnemannstraße 246 Miet- und 480 Eigentumswohnungen im ausschließlich gehobenen bis luxuriösen Marktsegment entstanden (Stadt Frankfurt 2015: 79 f.). Nördlich der Sonnemannstraße werden zwar in zwei Neubauprojekten insgesamt auch 274 Wohneinheiten im geförderten Wohnungsbau entweder als klassische Sozial- wohnungen im 1. Förderweg (5,00 bis 5,50 Euro/m²) oder im Rahmen von städtischen Mittelschichtprogrammen mit einkommensabhängiger Förderung errichtet (Magistrat der Stadt Frankfurt 2015: 3), auffällig ist jedoch, dass sämtliche geförderten Wohnungen bereits vor 2000 und damit in der frühen Phase der Sanierung entstehen (vgl. Abb. 4). Konkret werden 1994 zunächst 51 und 1998 weitere 40 Sozialwohnungen zwischen Howaldtstraße und Windeckstraße errichtet sowie 1999183 Wohneinheiten mit einkommensabhängiger Förderung zwischen Ostendstraße und MartinElsässer-Weg eingeweiht (Stadt Frankfurt 2015: 30, 35). Bei allen folgenden, viel umfangreicheren Neubauprojekten mit insgesamt über 700 Wohneinheiten war dagegen kein sozialer Wohnungsbau mehr vorgesehen (Stadt Frankfurt, Stadtplanungsamt 2007; Stadt Frankfurt 2015: 79 ff.). Von diesen 274 geförderten Wohneinheiten befanden sich laut Angaben des Wohnungsamtes Ende 2014 zudem nur noch 51 Sozialwohnungen in der Preis- und Belegungsbindung gemäß dem 1. Förderweg; und zwar jene, die bereits 1994 an der Howaldtstraße errichtet worden sind (vgl. die drei roten Punkte im Sanierungsgebiet in Abb. 4).

\subsection{Fehlende Maßnahmen zur effektiven Verhinderung von Verdrängungsprozessen}

Im Unterschied zum Programm der „Einfachen Stadterneuerung" werden die jüngeren Aufwertungsmaßnahmen im Rahmen der städtebaulichen Sanierung zwischen 2000 und 2015 also nicht mehr von Instrumenten flankiert, die der Verdrängung einkommensschwacher Schichten entgegenwirken. Stattdessen werden Flächen nun von gewinnorientierten Akteuren im freifinanzierten Neubau und völlig ohne sozialen Wohnungsbau entwickelt. Legitimiert wird dieser Wandel mit dem Entwicklungsziel, durch eine ,ausgewogene soziale Mischung“ die „einseitige und instabile“ Bevölkerungsstruktur des Viertels stabilisieren und „für einen sozialen Ausgleich" sorgen zu wollen (Stadt Frankfurt 2015: 38, 83 f.). Zwar wird Ende 2014 mit Geltung ab 2016 im Ostend eine Erhaltungssatzung nach Baugesetzbuch zur Verhinderung von Verdrängungsprozessen eingeführt, allerdings ist angesichts des beschränkten Kriterienkatalogs und der fehlenden Umwandlungsverordnung ihre tatsächliche Effektivität fraglich.

\subsubsection{Profitorientierter Wohnungsbau}

Bis Ende der 1990er-Jahre erfolgt der Neubau im Sanierungsgebiet vorrangig im Rahmen des sozialen Wohnungsbaus. Ab der Jahrtausendwende wird der Wohnungsbau im Ostend dagegen von frei finanziertem Neubau dominiert und von profitorientierten Investoren getragen. Stadtplanerisch werden beispielsweise keine Grundstücke für genossenschaftliche oder gemeinnützig ausgerichtete Wohnungs- 
bauträger mit potenziell vergleichsweise günstigen Mieten vorgehalten (Günther 2014: 38). ${ }^{15}$ Gegenwärtig wird dieser Politikwechsel am deutlichsten anhand der stadtplanerischen Erschließung der ehemaligen industriellen Brachflächen nördlich des Osthafens sichtbar, wo außerhalb des ehemaligen Sanierungsgebietes ab Sommer 2016 in mehreren Bauprojekten neben Hotels und Büroraum bis zu 1.000 weitere Wohneinheiten entstehen und wo die Stadt eigene Grundstücke besitzt (Günther 2014: 38). Obwohl hier neues Baurecht geschaffen wird, werden weder die sich daraus ergebenden baurechtlichen Eingriffsmöglichkeiten noch das öffentliche Eigentum an Grund und Boden seitens der Stadt genutzt, um bezahlbaren Wohnraum zu realisieren. Anwohner kritisieren vielmehr, dass , die Stadt ihre wenigen eigenen Grundstücke in Erbpacht an private Investoren vergibt, anstatt dort selbst günstigen Wohnraum zu schaffen" (Günther 2014: 38). All dies führt letztlich dazu, dass im Ostend nun Kaufpreise von 4.600 Euro/ $\mathrm{m}^{2}$ erzielt (Schulze 2015: 30) bzw. Mieten von bis zu 15,50 Euro/m² (Manus 2015) verlangt werden.

\subsubsection{Rückzug aus dem sozialen Wohnungsbau}

Seit Anfang der 1990er-Jahre ist der Bestand an Sozialwohnungen in Frankfurt von knapp 70.000 Wohneinheiten (= etwa $20 \%$ des Wohnbestandes) auf unter 30.000 (= etwa $8 \%$ des Wohnbestandes) zurückgegangen (Schipper 2013b: 350). Diese Entwicklung spiegelt sich auch im Ostend wider, da zwar noch bis Ende der 1990er-Jahre 274 geförderte Wohnungen im Sanierungsgebiet errichtet worden sind, der geförderte und mietpreisgebundene Wohnungsbau jedoch seit 2000 keine Rolle mehr spielt (vgl. Abb. 4). ${ }^{16}$ Von den insgesamt 16.368 Wohnungen im Stadtviertel unterlagen 2014 noch 886 bzw. 5,4\% des Gesamtbestandes der Sozialbindung gemäß dem 1. Förderweg (Stadt Frankfurt am Main, Amt für Wohnungswesen 2015: 70 ff.). ${ }^{17}$ Zurzeit deutet auch nichts darauf hin, dass sich diese Politik beispielsweise bezogen auf die umfangreichen Neubauprojekte im Bereich nördlich des Osthafens ändern wird. Zwar hat die Stadt Frankfurt in Anbetracht zunehmender sozialer

\footnotetext{
15 Auch die städtische Wohnungsbaugesellschaft ABG Holding wird im Ostend nicht aktiv. Aufgrund der gewinnorientierten Restrukturierung des ehemals gemeinnützigen öffentlichen Unternehmens seit Anfang der 1990er-Jahre wäre von diesem kommunalen Investor jedoch nicht zu erwarten gewesen, bezahlbaren Wohnraum in nennenswertem Umfang zu schaffen (Schipper/Wiegand 2015).

16 Als Alternative zum sozialen Wohnungsbau hat die Stadt Frankfurt seit 2007 begonnen, Belegungsrechte im Bestand für eine Dauer von 10 bis 15 Jahren anzukaufen. Angesichts des geringen Interesses privater Eigentümer konnten so im Ostend allerdings insgesamt nur 15 Wohneinheiten (Stand Ende 2014) in die Preis- und Belegungsbindung genommen werden (vgl. Abb. 4).

17 Darüber hinaus unterliegen weitere 192 Wohneinheiten einer Preisbindung gemäß dem Frankfurter Mittelschichtprogramm.
}

Proteste 2014 im Wohnbaulandentwicklungsplan angekündigt, dass bei Neubauprojekten zukünftig eine Quote von $30 \%$ gefördertem Wohnraum angestrebt wird (Magistrat der Stadt Frankfurt 2014a), allerdings ist diese Selbstverpflichtung als „Soll“- und nicht als „Muss“-Bestimmung formuliert und daher bislang auch noch bei kaum einem Bauprojekt erreicht worden (BBSR 2014: 27). ${ }^{18}$ Vielmehr nutzt die Stadt ihre selbst geschaffene Flexibilität, um insbesondere in attraktiven, innerstädtischen Lagen gegenüber Investoren auf sozialpolitische Vorgaben verzichten zu können (Göpfert 2016), auch im Ostend, etwa in dem genannten Bereich nördlich des Osthafens. Obwohl hier auf ehemaligen industriellen Brachflächen neues Baurecht für 1.000 Wohnungen geschaffen wird, hat die Stadt den privaten Investoren auch in Bezug auf Sozialwohnungen keinerlei Auflagen erteilt, dort belegungs- und preisgebundene Wohnungen zu errichten (Günther 2014). Kritik von Anwohnern und Stadtteilpolitikern aus dem Ortsbeirat, die angesichts des gehobenen Neubaus eine Verdrängung von Mietern und sozialen Einrichtungen befürchten, entgegnet das Stadtplanungsamt vielmehr, dass ,man die Investoren nicht über Gebühr belasten dürfe" (Günther 2014: 38) und deswegen bewusst keine geförderten Wohnungen vorgeschrieben habe.

\subsubsection{Milieuschutzsatzung}

Angesichts vermehrter Mieterproteste prüft die Stadt Frankfurt am Main ab Ende 2014, Milieuschutzsatzungen (Erhaltungssatzungen nach Baugesetzbuch), unter anderem für Teile des Ostends, „zur Dämpfung einer unerwünschten, übermäßigen Aufwertungs- und Verdrängungsdynamik“" (Magistrat der Stadt Frankfurt 2014b: 2) zu erlassen. Mit Geltung ab 2016 und einer zeitlichen Befristung auf fünf Jahre ist geplant, Luxussanierungen bzw. „die Aufwertungen von Wohnraum auf überdurchschnittlichen Standard“ (Magistrat der Stadt Frankfurt 2014b: 3) unter Genehmigungsvorbehalt zu stellen. Dazu zählt die Zusammenlegung von Wohnungen zu einer Großwohnung von mehr als $130 \mathrm{~m}^{2}$, Balkone größer als $8 \mathrm{~m}^{2}$ und Personenaufzüge, die nur die oberen Geschosse erschließen. Bauliche Modernisierungsmaßnahmen, die der Herstellung eines zeitgemäBen Ausstattungsstandards dienen, sind davon ausdrücklich ausgenommen. Darüber hinaus sind die Frankfurter Milieuschutzsatzungen nicht mit einem Umwandlungsverbot von Miet- in Eigentumswohnungen gekoppelt, da die hessische Landesregierung bislang keine entsprechende Rahmenverordnung erlassen hat. Ohne dieses entscheidende Instrument und angesichts des beschränkten Kriterienkatalogs bezüglich der Nichtzulässigkeit von baulichen

\footnotetext{
${ }_{18}$ Im Europaviertel, in dem etwa 6.000 neue Wohnungen entstehen, liegt diese Quote bei unter 7\% (Schipper/Wiegand 2015: 18 f.).
} 
Aufwertungsmaßnahmen beurteilen Mieterinitiativen die Einführung der Satzungen als Symbolpolitik, die nicht nur viel zu spät käme, sondern - im Gegensatz zu den Mietpreisbindungen der frühen 1990er-Jahre (vgl. Kap. 3.1) - auch relativ wirkungslos bliebe. Der Milieuschutz in Frankfurt sei, so etwa die Frankfurter Allgemeine Zeitung (Schulze 2014a: 29), ,Symbolpolitik mit hohem Aufwand und geringem Ertrag“.

\subsection{Zwischenfazit: Stadtpolitisch forcierte Aufwertungs- und Veränderungsprozesse}

Seit Ende der 1980er-Jahre wendet die Stadt Frankfurt am Main zahlreiche Instrumente an, um das Ostend als Wohnviertel und Dienstleistungsstandort aufzuwerten. Vor diesem Hintergrund ist seit Mitte der 2000er-Jahre eine Gentrifizierungsdynamik in Gang gekommen, die nun zu steigenden Mieten und Verdrängungsprozessen führt. Die erfolgreiche Aufwertung des Ostends ist jedoch kein Resultat einer linearen und bruchlosen Strategie. Vielmehr waren die frühen Maßnahmen bis etwa 1999 noch eng von Mechanismen begleitet, die der Verdrängung einkommensschwacher Schichten zumindest temporär entgegenwirkten; beispielsweise waren die öffentlichen Sanierungszuschüsse noch an Mietobergrenzen gebunden und es wurde Neubau noch prioritär als sozialer Wohnungsbau betrieben. Folglich waren die frühen Aufwertungsbestrebungen noch stark von Rationalitäten des sozialen Ausgleichs geprägt, indem durch begleitende wohnungspolitische Interventionen Marktkräfte im Interesse einkommensschwacher Schichten zumindest befristet außer Kraft gesetzt worden sind. Ein grundlegender Wandel hin zu einer tiefgreifenden Neoliberalisierung von Stadtentwicklungs- und Wohnungspolitik erfolgt im Ostend erst ab Anfang der 2000er-Jahre. Angesichts einer von Austerität geprägten Krise der Kommunalfinanzen, einer hegemonialen Akzeptanz von Markt- und Wettbewerbslogiken und einer damit verbundenen Neuordnung städtischer Politik in Frankfurt, die sich nun verstärkt an den (Wohn-)Bedürfnissen einkommensstarker Gruppen ausrichtet (Schipper 2013b), verschiebt sich auch der Charakter der Aufwertungsmaßnahmen im Ostend. Anders ausgedrückt erfolgte die Aufwertung seitdem marktkonform, das heißt konkret ohne flankierende Maßnahmen, die effektiv darauf abzielen, bezahlbaren Wohnraum für niedrige Einkommensgruppen zu sichern und Verdrängung zu verhindern. Die Einführung der Milieuschutzsatzung Anfang 2016 mag hier zwar ein erstes Umdenken seitens der städtischen Akteure andeuten, allerdings muss die zu erwartende Wirkung dieses Instruments relativiert werden, da ohne Umwandlungsverordnung und ohne strengere Vorgaben bezüglich der Zulässigkeit von Bauvorhaben Verdrängungsprozesse nur bedingt zu verhindern sind.

\section{Die Rolle der Europäischen Zentralbank in gegenwärtigen Gentrifizierungsprozessen}

„Der Osten der Stadt ändert sein Gesicht. Fast ein Dutzend Bauvorhaben werden in den nächsten Jahren verwirklicht. Das liegt auch an der EZB“" (Schulze 2014b: 37).

Nachdem Anfang der 1990er-Jahre Frankfurt den Zuschlag als zukünftigen Sitz der Europäischen Zentralbank erhält, bezieht die Bank zuerst ein Hochhaus im Zentrum des Bankenviertels. Da dieses Gebäude jedoch nicht dem wachsenden Raumbedarf entspricht, tritt die Europäische Zentralbank schon früh in Verhandlungen mit der Stadt, um an anderer Stelle einen repräsentativen Neubau errichten zu können. Seit Anfang der 2000er-Jahre rückt dabei die Großmarkthalle im Ostend in den Fokus, weil sich hier die Interessen der Bank an einem großflächigen, innerstädtischen Areal sowie die Bestrebungen der Stadt, das Ostend aufwerten zu wollen, treffen. Das Stadtplanungsamt (Stadt Frankfurt, Stadtplanungsamt 2007: 26) formuliert beispielsweise: „Die Entscheidung, den Sitz der EZB auf das Areal des früheren Großmarktes zu verlegen, gibt zugleich einen wichtigen Impuls für die weitere städtebauliche Entwicklung des Frankfurter Ostens und seine Aufwertung gegenüber dem Westen der Stadt". Auch vom Ortsbeirat Bornheim/Ostend (2001) kommt diesbezüglich kaum Widerspruch. Zwar wird kritisiert, dass die Stadtteilpolitiker lediglich aus der Presse von den Verhandlungen zwischen Stadt und Europäischer Zentralbank erfahren und auch im weiteren Verlauf nur schlecht informiert bleiben; jedoch „,begrüßt“ der Beirat es prinzipiell „sehr, wenn das Ostend durch neue Baumaßnahmen aufgewertet wird" (Ortsbeirat Bornheim/Ostend 2001: 1). Angesichts dieser Interessenskonstellation kann die Europäische Zentralbank schnell eine Einigung mit der Stadt erzielen und das entsprechende Grundstück bereits 2002 kaufen. Nach einer längeren Bauphase wird der 1,3 Mrd. Euro teure Neubau im November 2014 bezogen. Die offizielle, von heftigen Anti-Austeritäts-Protesten des BlockupyBündnisses begleitete Eröffnung erfolgt im März 2015.

Bemerkenswert ist, dass das Ostend bereits ab Anfang 2002, also kurz nachdem feststand, dass die Europäische Zentralbank ins Ostend zieht, verstärkt in den Fokus immobilienwirtschaftlicher Verwertungsstrategien gerät. So schrieb beispielsweise die Frankfurter Allgemeine Zeitung: „Ungeachtet der konjunkturellen Abkühlung an den Immobilienmärkten hegen etliche Investoren große Pläne für das Frankfurter Ostend. Spätestens seitdem sich die Europäische Zentralbank (EZB) entschieden hat, ihren Sitz auf dem Gelände des Großmarktes im Osten der Stadt zu errichten, ist der Stadtteil ins Blickfeld der Immobilienbranche gerückt" (Ochs 2002: 49). Ähnlich diagnostiziert auch Bischoff (2007: 204 f.), dass seitdem „,der Umzug der Europäischen Zentralbank (EZB) auf das Großmarkthallengelände beschlossen ist, die Interessengemeinschaft 
Eastside, ein Zusammenschluss von Immobilienkaufleuten im Ostend“ begonnen hat, sich ,mit einer gemeinsamen Vermarktung ihrer Immobilienprojekte in Szene“ zu setzen. Nachdem das Ostend jahrzehntelang von einer im stadtweiten Vergleich schwachen Bodenpreisdynamik gekennzeichnet war (vgl. Abb. 1), spiegelt sich dieser Wandel immobilienwirtschaftlicher Verwertungsstrategien ab Mitte der 2000er-Jahre auch in deutlichen Bodenpreissteigerungen wider (vgl. Abb. 2).

Kritiker, die die Bedeutung der Europäischen Zentralbank im Kontext gegenwärtiger Aufwertungsprozesse als überbewertet erachten, halten dem entgegen, dass die Bank keine Gentrifizierungsprozesse auslöse, weil ihre hochbezahlten Mitarbeiter über die ganze Stadt verteilt wohnen und daher die Nachfrage im Ostend nach gehobenem Wohnraum nicht zusätzlich anheizen würden (Schulze 2015). Der Einwand mag zwar sachlich zutreffend sein, da die Wohnungsnachfrage von Mitarbeitern der Europäischen Zentralbank im Ostend in der Tat wahrscheinlich eher zu vernachlässigen ist. Allerdings geht eine solche Kritik an den entscheidenden Wirkungsmechanismen vorbei, da nicht die direkte Nachfrage von Beschäftigten der Europäischen Zentralbank, sondern vielmehr die materielle sowie vor allem symbolische Aufwertung des Viertels durch den lokalräumlichen Umzug der Zentralbank ausschlaggebend sind. Auf materieller Ebene gehen beispielsweise zahlreiche Aufwertungsmaßnahmen im Stadtteil direkt auf konkrete Forderungen der Zentralbank zurück. Diese konnte im Rahmen der Umzugsverhandlungen mit der Stadt erfolgreich durchsetzen, dass der Danziger Platz umgestaltet, der Ostbahnhof grundlegend modernisiert, die Verkehrsinfrastruktur verbessert und eine neue Brücke über den Main errichtet wird (Nienhaus 2013).

Symbolisch galt das Ostend vor dem Umzug der Europäischen Zentralbank zudem trotz aller bisherigen Aufwertungs- und Sanierungsmaßnahmen noch immer als „Schmuddelkind der Stadt“ (Nienhaus 2013: 25). Die Trägheit des negativen Images, welches jahrzehntelang einen gewissen Schutz vor steigenden Mieten und Wohnungspreisen bot, wird erst mit der Ankündigung, den Neubau der Europäischen Zentralbank im Ostend zu errichten, endgültig durchbrochen. So schreibt etwa die Frankfurter Allgemeine Zeitung: „Nun wächst hier die neue Europäische Zentralbank in den Himmel. Und plötzlich wird es schick“ (Nienhaus 2013: 25). Für Projektentwickler aus der Immobilienbranche sorgt die symbolische Aufwertung des Stadtteils durch die Europäische Zentralbank laut eigener Aussage für Euphorie und eine „Boom“-Stimmung, da sich nun das Negativimage umkehre und der Stadtteil darüber hinaus auf (inter-)nationaler Ebene in den Fokus der öffentlichen Aufmerksamkeit gerate; etwa indem man nun regelmäßig ,in der Tagesschau das Ostend sehen“ (Schulze 2014b: 37) könne.
Der Neubau der Europäischen Zentralbank wirkt also lokalräumlich dahingehend, dass das Ostend einen radikalen Imagewandel erfährt und entsprechend leichter für Immobilienentwickler als gehobenes Wohnviertel zu vermarkten ist. Die symbolische Aufwertung führt darüber hinaus dazu, dass auf der Nachfrageseite einkommensstarke Haushalte im Allgemeinen (und nicht nur Beschäftigte der Europäischen Zentralbank) nun zunehmend bereit sind, für Wohnungen im Ostend höhere Mieten und Preise zu zahlen, was es wiederum Immobilieneigentümern erlaubt, sich über die Verwertung des gestiegenen Lagepotenzials ihrer Wohnungen und Grundstücke höhere Grundrenten anzueignen (Schipper 2013a). Die diskursive Repositionierung des Ostends hat also eine sich selbsterfüllende Prophezeiung in Gang gesetzt. Die durch den Umzug ausgelöste symbolische Aufwertung des Stadtteils resultiert in steigenden immobilienwirtschaftlichen Ertragserwartungen mit entsprechend spekulativen Preisentwicklungen - wodurch die symbolisch-diskursive Verschiebung letztlich performativ ihre Bestätigung erfährt. Makroökonomische Rahmenbedingung dafür ist jedoch, dass auf den globalen Kapitalmärkten die notwendigen Investitionssummen für Anlagen in Wohnimmobilien bereitstehen. Wie wir im folgenden Exkurs zeigen werden, ist auch diesbezüglich die Rolle der Europäischen Zentralbank nicht zu unterschätzen.

Exkurs: Expansive Geldpolitik und Immobilienmärkte Angesichts der Finanz- und Schuldenkrisen seit 2008, einer Überakkumulation von Kapital, sinkenden Profitraten und eben auch aufgrund der geldpolitischen Lockerung durch die Europäische Zentralbank fließt insbesondere seit 2010 anlagesuchendes Kapital in den Immobiliensektor bzw. sekundären Kapitalkreislauf (Harvey 1982) und hier verstärkt in den deutschen, als sicher und unterbewertet geltenden Wohnungsmarkt (Schönig 2013; Hiller 2014; Fehlberg/ Mießner 2015), wobei zuerst attraktive, innerstädtische Viertel in wachsenden Metropolregionen, wie etwa das Ostend, in den Fokus geraten sind (Heeg 2013).

Um die Auswirkungen der globalen Finanzkrise und der anschließenden europäischen Staatsschuldenkrise zu begrenzen, verfolgt die Europäische Zentralbank - ähnlich wie andere Notenbanken auch - seit 2009 eine expansive Geldpolitik. Ausgehend von den Erfahrungen aus der Weltwirtschaftskrise der 1930er-Jahre, wonach eine kontraktive Zentralbankpolitik krisenverschärfend wirkt, hat die Europäische Zentralbank den Leitzinssatz seit Oktober 2008 stufenweise von 4,25\% auf derzeit 0,05\% (Dezember 2015) abgesenkt und damit die Liquiditätszufuhr in das Bankensystem deutlich ausgeweitet (Brühl/Walz 2015: 8), um die Finanzstabilität in Europa zu sichern. Darüber hinaus hat die Europäische Zentralbank in jüngster Zeit weitere Maßnahmen getroffen in der Hoffnung, durch die Ausweitung des Kreditangebots Wachstum zu erzeugen. 
Dazu zählt etwa der seit Anfang 2015 bis mindestens März 2017 erfolgende Ankauf von Staatsanleihen und weiteren Vermögenswerten in Höhe von 60 Mrd. Euro monatlich (Allen 2015).

Seit Beginn der Krise hat die Europäische Zentralbank durch diese Maßnahmen zwar ihren Bilanzumfang verdreifacht, allerdings ist es ihr mittels der Niedrigzinspolitik und der Wertpapieraufkäufe nicht gelungen, substanzielle Wachstumsimpulse zu setzen. Vielmehr weisen Kader und Schwarzer (2015: $289 \mathrm{ff}$.) darauf hin, dass Investitionen und Konsumausgaben nicht in einem relevanten Ausmaß gestiegen seien, sondern die expansive Geldpolitik lediglich die Inflation von Aktien, Anleihen und Immobilienpreisen antreibe. Dies habe vor allem den Effekt, dass die Vermögen der besitzenden Klasse gesteigert würden und sich die soziale Ungleichheit verschärfe.

Unabhängig von der kontrovers diskutierten Frage, ob sich hinter der jüngsten Preisexplosion eine Blasenbildung am Immobilienmarkt abzeichnet, besteht innerhalb der wirtschaftswissenschaftlichen Diskussion ein breiter Konsens darüber, dass die expansive Geldpolitik der Europäischen Zentralbank strukturell ebenso für den jüngsten Preisanstieg am Wohnungsmarkt mitverantwortlich ist (Hiller 2014; Brühl/Walz 2015; Kholodilin/Michelsen/Ulbricht 2014). Bezogen auf den Immobilienmarkt in Deutschland hat die Kreditmengenexpansion zum Beispiel dazu geführt, dass die Preise für deutsche Wohnimmobilien seit $2010 \mathrm{im}$ Durchschnitt um mehr als $10 \%$ gestiegen sind (Brühl/Walz 2015: 27). Hinter solchen Durchschnittswerten verbergen sich aber regional zutiefst ungleiche Entwicklungen (Heeg 2013), da beispielsweise in Frankfurt am Main im gleichen Zeitraum (2010-2014) die Wohnungspreise im Neubau um $40 \%$ (Gutachterausschuss für Immobilienwerte 2014: 22) und die Angebotsmieten jährlich im Durchschnitt um knapp $4 \%$ angewachsen sind (IWU 2015: 50). Zusammengefasst trägt also auch die expansive Kreditmengenausweitung dazu bei, dass Kapital in den Immobiliensektor und dort insbesondere in innerstädtische Viertel mit Aufwertungspotenzial, wie etwa dem Ostend, fließt und Gentrifizierungsprozesse antreibt.

\section{Zusammenfassung}

Während das Ostend als klassisches Arbeiterviertel jahrzehntelang vergleichsweise bezahlbaren Wohnraum für untere Einkommensgruppen bot, zeichnet sich ab Mitte der 2000er-Jahre ein grundlegender Wandel des Stadtteils ab. Nach Davidson und Lees (2005: 1187) kann dieser Wandel als Gentrifizierung bezeichnet werden, weil erstens Haushalte mit niedrigen Einkommen von exkludierender Verdrängung betroffen sind, zweitens der Zuzug einkommensstarker Gruppen die Bevölkerungsstruktur im Viertel ver- schiebt, sich drittens die urbane Landschaft tiefgreifend wandelt und viertens verstärkt anlagesuchendes Kapital in die gebaute Umwelt fließt. Direkte Verdrängung lässt sich nicht explizit nachweisen, allerdings ein langfristiger soziostruktureller Wandel, der zur Folge hat, dass heute Sozialleistungsbezug und Arbeitslosigkeit im gesamtstädtischen Vergleich unter- und die Einkommen überdurchschnittlich sind. Im Unterschied zu den Phasenmodellen der Gentrifizierung (vgl. Friedrichs 1996), in denen der Wandel häufig durch eine Phase als Szeneviertel eingeläutet wird, lässt sich im Ostend kein derartiges Stadium ausmachen. Auch die in diesen Modellen wichtige Akteursgruppe der Pioniere spielt im Ostend keine bedeutende Rolle.

Angestoßen und gefördert worden sind diese Transformationsprozesse von stadtpolitischen Aufwertungsstrategien, deren Ursprung sich bis in die späten 1980er-Jahre zurückverfolgen lässt. Auch wenn die Aufwertung des Viertels demnach schon seit Jahrzehnten auf der stadtpolitischen Agenda steht, waren die entsprechenden Maßnahmen allerdings noch bis Ende der 1990er-Jahre von Mechanismen begleitet, die die Verdrängung einkommensschwacher Schichten verhindern oder zumindest bremsen sollten. Seit der Jahrtausendwende erfolgt die Aufwertung jedoch marktkonform, was folglich Verdrängungsprozesse beschleunigt.

In Kap. 4 konnten wir darüber hinaus zeigen, dass auch die Europäische Zentralbank die Gentrifizierung im Ostend vorantreibt. Der Umzug und Neubau des Zentralbanksitzes hat eine grundlegende symbolisch-diskursive Repositionierung des Viertels bewirkt, was entscheidend dazu beiträgt, dass sich das negative und lange Zeit preisdämpfend wirkende Image des Stadtteils radikal wandelt. Die damit einhergehende Steigerung des Lagepotenzials ermöglicht es den Eigentümern von Immobilien nun aufgrund ihrer neu gewonnenen Monopolstellung (prestigeträchtige Nähe zur Europäischen Zentralbank), höhere Preise und Mieten zu verlangen und sich damit entsprechend hohe Grundrenten anzueignen. Dieser Mechanismus gewinnt insbesondere seit der globalen Finanzkrise von 2008 an Bedeutung, da Wohnraum auch angesichts der expansiven Geldpolitik der Europäischen Zentralbank als Anlagesphäre im Vergleich zu konkurrierenden Investitionsfeldern attraktive Renditen verspricht.

Um derartige Prozesse der Gentrifizierung zu bremsen, wäre, wie Kap. 3 gezeigt hat, zuallererst ein grundlegender wohnungspolitischer Paradigmenwechsel notwendig hin zur Zielsetzung, Verdrängung verhindern und bezahlbaren Wohnraum für Menschen mit geringen und mittleren Einkommen sicherstellen zu wollen. In Anbetracht der Schwäche bestehender stadtplanerischer Instrumente, wie etwa den 2016 eingeführten Milieuschutzsatzungen, wären zudem neue Strategien zu verfolgen, die die Wohnraumversorgung, etwa über kommunale, nichtgewinnorientierte Wohnungsunternehmen oder neue gemeinnützige Woh- 
nungsbauträger, dauerhaft dem Marktdruck und Prozessen der Finanzialisierung (Heeg 2013) entziehen.

\section{Literatur}

Allen, K. (2015): QE feeding Europe house price bubble, study says. In: Financial Times vom 20. Juli 2015. http://www.ft.com/cms/s/ 0/739a3700-2eeb-11e5-8873-775ba7c2ea3d.html (03.08.2016).

BBSR - Bundesinstitut für Bau-, Stadt- und Raumforschung (2014): Kommunale Strategien für die Versorgung einkommensschwächerer und sozial benachteiligter Haushalte. Bonn.

Bischoff, W. (2007): Nicht-visuelle Dimensionen des Städtischen. Olfaktorische Wahrnehmung in Frankfurt am Main, dargestellt an zwei Einzelstudien zum Frankfurter Westend und Ostend. Oldenburg. = Wahrnehmungsgeographische Studien 23.

Brede, H.; Dietrich, B.; Kohaupt, B. (1976): Politische Ökonomie des Bodens und Wohnungsfrage. Frankfurt am Main.

Brühl, V.; Walz, U. (2015): Das anhaltende Niedrigzinsumfeld in Deutschland. Frankfurt am Main. $=$ CFS Working Paper Series 506.

Clark, E. (1995): The Rent Gap Re-examined. In: Urban Studies 32, 9, 1489-1503.

Davidson, M.; Lees, L. (2005): New-build 'gentrification' and London's riverside renaissance. In: Environment and Planning A 37, 7, 1165-1190.

Fehlberg, T.; Mießner, M. (2015): Mietpreissteigerungen und Wohnungsengpässe abseits der Ballungsräume. Investitionen in Wohnimmobilien in B-Lagen und Regionalzentren - das Beispiel Göttingen. In: sublurban. zeitschrift für kritische stadtforschung $3,1,25-44$.

Freund, B. (2002): Hessen. Gotha, Stuttgart.

Friedrichs, J. (1996): Gentrification: Forschungsstand und methodologische Probleme. In: Friedrichs, J.; Kecskes, R. (Hrsg.): Gentrification. Theorie und Forschungsergebnisse. Opladen, 13-40.

Göpfert, C.-J. (2016): Luxuszone Frankfurter Innenstadt. In: Frankfurter Rundschau vom 10. März 2016. http://www.fr-online. de/stadtentwicklung/innenstadt-frankfurt-luxuszone-frankfurterinnenstadt, 26042926,33935592.html (02.08.2016).

Günther, B. (2014): Ostend. Hier sollten nicht nur Reiche wohnen. In: Frankfurter Allgemeine Zeitung vom 6. November 2014, Nr. 258, 38.

Gutachterausschuss für Immobilienwerte (2014): Immobilienmarktbericht für den Bereich Frankfurt am Main. Frankfurt am Main.

Harvey, D. (1982): The limits to capital. Oxford.

Heeg, S. (2013): Wohnungen als Finanzanlage. Auswirkungen von Responsibilisierung und Finanzialisierung im Bereich des Wohnens. In: sublurban. zeitschrift für kritische stadtforschung 1, 1, 75-99.

Hiller, N. (2014): Zinspolitik ade! Wie man Immobilienpreisblasen dennoch überstehen kann. In: Wirtschaftsdienst 94, 10, 748-755.

Holm, A. (2014): Gentrifizierung - mittlerweile ein Mainstreamphänomen? In: Informationen zur Raumentwicklung 4, 277-289.

IWU - Institut für Wohnen und Umwelt (2015): Feststellung von Gebieten mit angespannten Wohnungsmärkten im Sinne des § 556d Abs. 1 BGB anhand geeigneter Indikatoren im Land Hessen. Eine Untersuchung im Auftrag des Hessischen Ministeriums für Umwelt, Klimaschutz, Landwirtschaft und Verbraucherschutz. Darmstadt. https://umweltministerium.hessen.de/sites/ default/files/media/hmuelv/gutachten_neuvertragsmietbremse. pdf (26.07.2016).

Kader, M.; Schwarzer, U. (2015): Akkumulation reloaded. Wie Notenbanken und Staaten die Profite retten. In: Prokla. Zeitschrift für kritische Sozialwissenschaft 45, 2, 277-296.

Kholodilin, K. A.; Michelsen, C.; Ulbricht, D. (2014): Speculative price bubbles in urban housing markets in Germany. Berlin. = DIW Discussion Papers 1417.

Köneke, J. (2015): Viertel rund um die EZB wandelt sich. In: Frankfurter Rundschau vom 17. Januar 2015. http://www.fr-online.de/ frankfurt/ostend-viertel-rund-um-die-ezb-wandelt-sich, 1472798 , 29595784.html (26.07.2016).

Magistrat der Stadt Frankfurt (2003): Vortrag des Magistrats vom 06.06.2003, M 77. http://www.stvv.frankfurt.de/download/M 77_2003.pdf (26.07.2016).

Magistrat der Stadt Frankfurt (2014a): Vortrag des Magistrats vom 17.01.2014, M 9. http://www.stvv.frankfurt.de/download/M_9_ 2014.pdf (26.07.2016).

Magistrat der Stadt Frankfurt (2014b): Vortrag des Magistrats vom 15.12.2014, M 217. http://www.stvv.frankfurt.de/download/M 217_2014.pdf (26.07.2016).

Magistrat der Stadt Frankfurt (2015): Vortrag des Magistrats vom 18.05.2015, M 79. http://www.stvv.frankfurt.de/download/M_ 79_2015.pdf (26.07.2016).

Manus, C. (2015): Neues Hotel am Danziger Platz. In: Frankfurter Rundschau vom 30. September 2015. http://www.fr-online. de/stadtentwicklung/frankfurt-ostend-neues-hotel-am-danzigerplatz, 26042926,32051278.html (02.08.2016).

Marcuse, P. (1986): Abandonment, Gentrification, and Displacement: The Linkages in New York City. In: Smith, N.; Williams, P. (Hrsg.): Gentrification of the City. Boston, 153-177.

Nienhaus, L. (2013): Tagelöhner trifft Notenbanker. In: Frankfurter Allgemeine Sonntagszeitung vom 25. August 2013, Nr. 34, 25.

Ochs, B. (2002): Frankfurts Ostend zieht Investoren an. In: Frankfurter Allgemeine Zeitung vom 4. Januar 2002, Nr. 3, 49.

Ortsbeirat Bornheim/Ostend (2001): Antrag vom 15.01.2001, OF 591/4. http://www.stvv.frankfurt.de/download/OF_591-4_2001. pdf (26.07.2016).

Ronneberger, K.; Keil, R. (1995): Ausser Atem - Frankfurt nach der Postmoderne. In: Hitz, H.; Keil, R.; Lehrer, U.; Ronneberger, K.; Schmid, C.; Wolff, R. (Hrsg.): Capitales Fatales. Urbanisierung und Politik in den Finanzmetropolen Frankfurt und Zürich. Zürich, 286-353.

Schipper, S. (2013a): Global-City-Formierung, Gentrifizierung und Grundrentenbildung in Frankfurt am Main. In: Zeitschrift für Wirtschaftsgeographie 57, 4, 185-200.

Schipper, S. (2013b): Genealogie und Gegenwart der unternehmerischen Stadt. Neoliberales Regieren in Frankfurt am Main, 1960-2010. Münster.

Schipper, S.; Wiegand, F. (2015): Neubau-Gentrifizierung und globale Finanzkrise. Der Stadtteil Gallus in Frankfurt am Main zwischen immobilienwirtschaftlichen Verwertungszyklen, stadtpolitischen Aufwertungsstrategien und sozialer Verdrängung. In: sublurban. zeitschrift für kritische stadtforschung 3, 3, 7-32.

Schönig, B. (2013): Die neue Wohnungsfrage. In: Blätter für deutsche und internationale Politik 2, 17-20.

Schulze R. (2014a): Frankfurt will Milieus schützen. In: Frankfurter Allgemeine Zeitung vom 14. November 2014, Nr. 265, 29.

Schulze, R. (2014b): Das Frankfurter Ostend wird umgebaut. In: Frankfurter Allgemeine Zeitung vom 30. August 2014, Nr. 201, 37.

Schulze, R. (2015): Wie sich das Ostend wandelt. In: Frankfurter Allgemeine Zeitung vom 18. März 2015, Nr. 65, 30.

Smith, N. (1996): The new urban frontier. Gentrification and the revanchist city. London.

Stadt Frankfurt, Amt für kommunale Gesamtentwicklung und Stadtplanung (1994): Einfache Stadterneuerung Frankfurt am Main Ostend. Frankfurt am Main.

Stadt Frankfurt, Stadtplanungsamt (2007): Stadtumbau Frankfurter Osten. Frankfurt am Main.

Stadt Frankfurt, Stadtplanungsamt (2015): Abschlussbericht zur Sanierungsmaßnahme Frankfurt am Main ,Ostendstraße‘. Frankfurt am Main.

Stadt Frankfurt am Main, Amt für Wohnungswesen (2014): Mietspiegel 2014. Frankfurt am Main.

Stadt Frankfurt am Main, Amt für Wohnungswesen (2015): Tätigkeitsbericht 2014. Frankfurt am Main. http://www.frankfurt.de/ sixcms/media.php/738/t\%C3\%A4tigkeitsbericht2014.pdf (26.07.2016). 